\title{
Estimation and Testing Procedures for the Reliability Characteristics of Chen Distribution Based on Type II Censoring and the Sampling Scheme of Bartholomew
}

\author{
Aditi Chaturvedi *, Surinder Kumar \\ Department of Statistics, Babasaheb Bhimrao Ambedkar University, Lucknow, India
}

\begin{abstract}
In this paper, we consider Chen distribution and derive UMVUEs and MLEs of the parameter $\lambda$, hazard rate $h(t)$ and the two measures of reliability, namely $R(t)=P(X>t)$, where $X$ denotes the lifetime of an item and $P=P(X>Y)$, which represents the reliability of an item or system of random strength $X$ subject to random stress $Y$, under type II censoring scheme and the sampling scheme of Bartholomew. We also develop interval estimates of the reliability measures. Testing procedures for the hypotheses related to different parametric functions have also been developed. A comparative study of different methods of point estimation and average confiddence length has been done through simulation studies. The analysis of a real data set is presented for illustration purpose.
\end{abstract}

Keywords Chen Distribution, Hazard Rate, Interval Estimation, MLE, Monte Carlo Simulation, Point Estimation, UMVUE

AMS 2010 subject classifications 62N05, 62F10

DOI:10.19139/soic-2310-5070-1032

\section{Introduction}

In the reliability literature, we have many such distributions (e.g. generalized exponential, gamma, Weibull and lognormal) whose hazard rate functions are constant, increasing or decreasing in nature. These are the most commonly used models and we analyze various real life phenomenon using them. However, these models are not suitable if the data sets exhibit bathtub-shaped hazard rate. Authors have introduced some probability models to analyze real data with bathtub-shaped failure, for instance, modified Weibull [13] and extended Weibull [16], but still they are not suitable to produce a good bathtub shape of the failure rates.

[5] introduced a two-parameter lifetime distribution with bathtub shape or increasing failure rate function. The hazard rate of this distribution first decreases, then remains constant and then increases. Chen distribution is an appropriate model for analysis of electronic and mechanical products and lifetime of humans. Further, it can be used for modelling positively skewed data, apart from the well known models such as lognormal and gamma. This distribution is flexible in nature in the sense that it has two parameters and the confidence intervals for the shape parameter as well as the joint confidence regions have the closed form.

A random variable (rv) $X$ is said to follow the Chen distribution, if its probability density function (pdf) is of the form:

$$
f(x ; \lambda, \beta)=\lambda \beta e^{x^{\beta}} x^{\beta-1} e^{\lambda\left(1-e^{x^{\beta}}\right)},
$$

\footnotetext{
${ }^{*}$ Correspondence to: Aditi Chaturvedi (Email: caditic@gmail.com). Department of Statistics, Babasaheb Bhimrao Ambedkar University, Lucknow, Uttar Pradesh, India
}

ISSN 2310-5070 (online) ISSN 2311-004X (print)

Copyright (C) 2021 International Academic Press 
and cumulative distribution function (cdf) is of the form:

$$
F(x)=1-\exp \left[\lambda\left(1-e^{x^{\beta}}\right)\right] ; x>0 .
$$

Moreover, the hazard rate $h(t)$ of the distribution (1) corresponding to time ' $t$ ' is given by:

$$
h(t)=\lambda \beta t^{\beta-1} e^{t^{\beta}} ; t>0 .
$$

[21] obtained MLEs of the unknown parameters $\beta$ and $\lambda$ of the distribution (1) based on progressive censoring and also discussed the problem of interval estimation. [18] considered the Bayesian estimation for different symmetric and asymmetric loss functions. [1] proposed Bayes estimates of unknown parameters $\beta$ and $\lambda$ under balanced squared-error loss function. Bayesian estimation for the discrete Chen distribution was discussed by [12]. The recurrence relations for single and product moments of generalized order statistics from Chen distribution was established by [11]. [10] have obtained one-sample and two-sample Bayes predictive estimates and also constructed prediction intervals of censored observations under progressive censoring. Moreover, [9] have considered the estimation of unknown parameters $\beta$ and $\lambda$ using both classical and Bayesian approaches under type I progressive hybrid censoring scheme. They have considered the problem of optimal censoring as well. [8] have considered the problem of estimating the reliability in a multicomponent stress-strength model based on Chen distribution. [2] obtained Empirical Bayes estimators of the scale parameter, reliability and hazard rate functions of Chen distribution under the condition when a sample is obtained from a type-I censoring scheme.

The literature on estimation procedures for Chen distribution discussed above mostly focuses on maximum likelihood or Bayesian/empirical Bayesian procedures. However, developing and investigating properties of UMVU estimators for the parameters of Chen distribution under various sampling schemes is an area which still remain unexplored. The present work is an attempt to fill this gap. The objective is to develop point estimation procedures for $R(t)$ and $P$ based on type II censoring and the sampling scheme of Bartholomew [3] with the help of a technique proposed by [4] which is simpler and not time consuming. In this technique we first obtain the estimator of the powers of parameter $\lambda$ and then with the help of this estimator we obtain estimator of the pdf. The estimator of the pdf is further used to obtain estimator of $R(t)$ and $P$. The paper is organized as follows: In Section 2, we provide MLEs and UMVUEs of parameter $\lambda^{q}$, hazard rate $h(t)$ and the reliability functions $R(t)$ and $P$ based on type II censoring scheme assuming $\beta$ to be known. We also provide exact confidence intervals for $\lambda, R(t)$ and $P$. Further, we develop testing procedures for $\lambda$, when $\beta$ is known. In Section 3, we obtain MLEs and UMVUEs of $\lambda^{q}$, hazard rate $h(t)$ and the reliability functions $R(t)$ and $P$ based on the censoring scheme of Bartholomew assuming $\beta$ to be known. We also provide testing procedures for $\lambda$ based on this censoring scheme for known $\beta$ case. In Section 4, we provide extensive sets of simulation studies followed by a real data example in Section 5 . We end with a brief set of conclusions in Section 6. Proofs of some important results can be found in the Appendix.

\section{Estimation and Testing Procedures Based on Type II Censoring Scheme}

Suppose $n$ items are put on a test and the test is terminated after the first $r$ ordered observations are recorded. Let us denote by $0<X_{(1)} \leq X_{(2)} \leq \ldots \leq X_{(r)}, 0<r<n$, the lifetimes of first $r$ failures. Obviously, $(n-r)$ items survived until $X_{(r)}$.

\subsection{UMVUE's and MLE's of $\lambda^{q}, R(t), P$ and $h(t)$}

In this section, we obtain the UMVUE's and MLE's of $\lambda^{q}, R(t), P$ and $h(t)$ under the assumption that $\beta$ is known. We first provide an important lemma, which will be useful in proving the main results of this section.

\section{Lemma 1}

Let

$$
S_{(r)}=\sum_{i=1}^{r}\left(e^{x_{(i)}^{\beta}}-1\right)+(n-r)\left(e^{x_{(r)}^{\beta}}-1\right) .
$$


Then, $S_{(r)}$ is complete and sufficient for the distribution given at (1). Moreover, the pdf of $S_{r}$ is given by

$$
g_{S_{(r)}}(s ; \lambda)=\frac{s^{r-1} \lambda^{r} e^{-\lambda s}}{\Gamma(r)}, s>0, \alpha>0, r>0,
$$

where, $\Gamma(\cdot)$ denotes the Gamma function.

Proof The proof of Lemma 1 can be found in the Appendix.

The pdf of $S_{(r)}$ given in (4) can be used to obtain the UMVUE of $\lambda^{q}$. In this direction, we have from (4)

$$
\begin{aligned}
E\left(S_{(r)}^{-q}\right) & =\frac{\lambda^{r}}{\Gamma(r)} \int_{0}^{\infty} e^{-\lambda s} s^{r-q-1} d s \\
& =\frac{\Gamma(r-q)}{\Gamma(r)} \lambda^{q}, r>q .
\end{aligned}
$$

Now using the Lehmann-Scheffe theorem (see, [19]), for $q \in(-\infty, \infty)$, the UMVUE of $\lambda^{q}$ is given by

$$
\widetilde{\lambda}_{I I}^{q}= \begin{cases}\frac{\Gamma(r)}{\Gamma(r-q)} S_{(r)}^{-q} & : r-q>0 \\ 0 & : \text { otherwise }\end{cases}
$$

Further, we can write the pdf given in (1) as

$$
f(x ; \lambda, \beta)=\lambda \beta e^{x^{\beta}} x^{\beta-1} \sum_{i=0}^{\infty} \frac{(-1)^{i}}{i !}\left\{\lambda\left(e^{x^{\beta}}-1\right)\right\}^{i}
$$

Making use of the UMVUE of $\lambda^{q}$ given in (5), the UMVUE of the sampled pdf at a specified point ' $x$ ' is given by

$$
\begin{aligned}
\widetilde{f}_{I I}(x ; \lambda, \beta) & =\beta e^{x^{\beta}} x^{\beta-1} \sum_{i=0}^{\infty} \frac{(-1)^{i}}{i !}\left(e^{x^{\beta}}-1\right)^{i} \widetilde{\lambda^{i+1}} \\
& =\beta e^{x^{\beta}} x^{\beta-1} \sum_{i=0}^{\infty} \frac{(-1)^{i}}{i !}\left(e^{x^{\beta}}-1\right)^{i} \frac{\Gamma(r)}{\Gamma(r-i-1)} S_{(r)}^{-(i-1)}
\end{aligned}
$$

which can further be written as

$$
\widetilde{f}_{I I}(x ; \lambda, \beta)= \begin{cases}(r-1) \frac{e^{x^{\beta}} \beta x^{\beta-1}}{S_{(r)}}\left(1-\frac{e^{x^{\beta}}-1}{S_{(r)}}\right)^{r-2} & : x<\left\{\ln \left(1+S_{(r)}\right)\right\}^{1 / \beta} \\ 0 & : \text { otherwise }\end{cases}
$$

Using the result given in (6), we can obtain the UMVUE of $R(t)$ as

$$
\begin{aligned}
\widetilde{R}(t)_{I I} & =\int_{t}^{\infty} \widetilde{f}(x ; \lambda, \beta) d x \\
& =\int_{t}^{\left(\ln \left(1+S_{(r)}\right)\right)^{1 / \beta}}(r-1) \frac{e^{x^{\beta}} \beta x^{\beta-1}}{S_{(r)}}\left(1-\frac{e^{x^{\beta}}-1}{S_{(r)}}\right)^{r-2} d x
\end{aligned}
$$

Substituting $\left(e^{x^{\beta}}-1\right) / S_{(r)}=y$ in the above expression, the UMVUE of $R(t)$ at a specified point ' $t$ ' is given by

$$
\widetilde{R}(t)_{I I}= \begin{cases}\left(1-\frac{e^{t^{\beta}}-1}{S_{(r)}}\right)^{r-1} & : e^{t^{\beta}}-1<S_{(r)} \\ 0 & : \text { otherwise }\end{cases}
$$


Let $X$ and $Y$ be two independent random variables following the classes of distributions $f_{1}\left(x ; \lambda_{1}, \beta_{1}\right)$ and $f_{2}\left(y ; \lambda_{2}, \beta_{2}\right)$, respectively, where

$$
\begin{aligned}
& f_{1}\left(x ; \lambda_{1}, \beta_{1}\right)=\lambda_{1} \beta_{1} e^{x^{\beta_{1}}} x^{\beta_{1}-1} e^{\lambda_{1}\left(1-e^{x^{\beta_{1}}}\right)} ; x>0, \lambda_{1}, \beta_{1}>0, \\
& f_{2}\left(y ; \lambda_{2}, \beta_{2}\right)=\lambda_{2} \beta_{2} e^{y^{\beta_{2}}} y^{\beta_{2}-1} e^{\lambda_{2}\left(1-e^{y_{2}}\right)} ; y>0, \lambda_{2}, \beta_{2}>0 .
\end{aligned}
$$

Let $n$ items on $X$ and $m$ items on $Y$ are put on a life test and the termination numbers for $X$ and $Y$ are $r$ and $r^{\prime}$, respectively. Let us define

$$
\begin{gathered}
S_{(r)}=\sum_{i=1}^{r}\left(e^{x_{(i)}^{\beta_{1}}}-1\right)+(n-r)\left(e^{x_{(r)}^{\beta_{1}}}-1\right), \\
T_{\left(r^{\prime}\right)}=\sum_{j=1}^{r^{\prime}}\left(e^{y_{(j)}^{\beta_{2}}}-1\right)+\left(m-r^{\prime}\right)\left(e^{y_{\left(r^{\prime}\right)}^{\beta_{2}}}-1\right) .
\end{gathered}
$$

It follows from (6) that, the UMVUE's of $f_{1}\left(x ; \lambda_{1}, \beta_{1}\right)$ and $f_{2}\left(y ; \lambda_{2}, \beta_{2}\right)$ based on Type II censoring at specified points $x$ and $y$, respectively, are given by

$$
\begin{aligned}
& \widetilde{f}_{1 I I}\left(x ; \lambda_{1}, \beta_{1}\right)=(r-1) \frac{e^{x^{\beta_{1}}} \beta_{1} x^{\beta_{1}-1}}{S_{(r)}}\left(1-\frac{e^{x^{\beta_{1}}}-1}{S_{(r)}}\right)^{r-2} ; x<\left\{\ln \left(1+S_{(r)}\right)\right\}^{1 / \beta_{1}}, \\
& \widetilde{f}_{2 I I}\left(y ; \lambda_{2}, \beta_{2}\right)=\left(r^{\prime}-1\right) \frac{e^{y^{\beta_{2}}} \beta_{2} y^{\beta_{2}-1}}{T_{\left(r^{\prime}\right)}}\left(1-\frac{e^{y^{\beta_{2}}}-1}{T_{\left(r^{\prime}\right)}}\right)^{r^{\prime}-2} ; y<\left\{\ln \left(1+T_{\left(r^{\prime}\right)}\right)\right\}^{1 / \beta_{2}} .
\end{aligned}
$$

The UMVUE of $P$ can be written in terms of $\widetilde{R}\left(y ; \lambda_{1}, \beta_{1}\right)_{I I}$ as follows:

$$
\begin{aligned}
\widetilde{P}_{I I} & =\int_{y=0}^{\infty} \int_{x=y}^{\infty} \widetilde{f}_{I I}\left(x ; \lambda_{1}, \beta_{1}\right) \tilde{f}_{I I}\left(y ; \lambda_{2}, \beta_{2}\right) d x d y \\
& =\int_{y=0}^{\infty} \widetilde{R}_{I I}\left(y ; \lambda_{1}, \beta_{1}\right) \widetilde{f}_{I I}\left(y ; \lambda_{2}, \beta_{2}\right) d y
\end{aligned}
$$

which on using (7) and (11) gives that

$$
\begin{aligned}
& \widetilde{P}_{I I}= \int_{y=0}^{\infty}\left(1-\frac{e^{y^{\beta_{1}}}-1}{S_{(r)}}\right)^{r-1}\left(r^{\prime}-1\right) \frac{e^{y^{\beta_{2}}} \beta_{2} y^{\beta_{2}-1}}{T_{\left(r^{\prime}\right)}}\left(1-\frac{e^{y^{\beta_{2}}}-1}{T_{\left(r^{\prime}\right)}}\right)^{r^{\prime}-2} d y, \\
& \text { where } y<\left\{\ln \left(1+S_{(r)}\right)\right\}^{1 / \beta_{1}}, y<\left\{\ln \left(1+T_{\left(r^{\prime}\right)}\right)\right\}^{1 / \beta_{2}}, \\
&=\int_{y=0}^{c^{\prime}}\left(1-\frac{e^{y^{\beta_{1}}}-1}{S_{(r)}}\right)^{r-1}\left(r^{\prime}-1\right) \frac{e^{y^{\beta_{2}}} \beta_{2} y^{\beta_{2}-1}}{T_{\left(r^{\prime}\right)}}\left(1-\frac{e^{y^{\beta_{2}}}-1}{T_{\left(r^{\prime}\right)}}\right)^{r^{\prime}-2} d y, \\
& \text { where } c^{\prime}=\min \left[\left\{\ln \left(1+S_{(r)}\right)\right\}^{1 / \beta_{1}},\left\{\ln \left(1+T_{\left(r^{\prime}\right)}\right)\right\}^{1 / \beta_{2}}\right] .
\end{aligned}
$$

From (12), for $\left\{\ln \left(1+S_{(r)}\right)\right\}^{1 / \beta_{1}}<\left\{\ln \left(1+T_{\left(r^{\prime}\right)}\right)\right\}^{1 / \beta_{2}}$, we have

$$
\widetilde{P}_{I I}=\int_{y=0}^{\left\{\ln \left(1+S_{(r)}\right)\right\}^{1 / \beta_{1}}}\left(1-\frac{e^{y^{\beta_{1}}}-1}{S_{(r)}}\right)^{r-1}\left(r^{\prime}-1\right) \frac{e^{y^{\beta_{2}}} \beta_{2} y^{\beta_{2}-1}}{T_{\left(r^{\prime}\right)}}\left(1-\frac{e^{y^{\beta_{2}}}-1}{T_{\left(r^{\prime}\right)}}\right)^{r^{\prime}-2} d y
$$


and for $\left\{\ln \left(1+S_{(r)}\right)\right\}^{1 / \beta_{1}} \geq\left\{\ln \left(1+T_{\left(r^{\prime}\right)}\right)\right\}^{1 / \beta_{2}}$, we have

$$
\widetilde{P_{I I}}=\int_{y=0}^{\left\{\ln \left(1+T_{\left(r^{\prime}\right)}\right)\right\}^{1 / \beta_{2}}}\left(1-\frac{e^{y^{\beta_{1}}}-1}{S_{(r)}}\right)^{r-1}\left(r^{\prime}-1\right) \frac{e^{y^{\beta_{2}}} \beta_{2} y^{\beta_{2}-1}}{T_{\left(r^{\prime}\right)}}\left(1-\frac{e^{y^{\beta_{2}}}-1}{T_{\left(r^{\prime}\right)}}\right)^{r^{\prime}-2} d y
$$

Substituting $\left(e^{y^{\beta_{2}}}-1\right) / T_{\left(r^{\prime}\right)}=z$ in (13) and $\left(e^{y^{\beta_{2}}}-1\right) / T_{\left(r^{\prime}\right)}=z$ in (14), we obtain the UMVUE of $P$ as

$$
\widetilde{P}_{I I}=\left\{\begin{array}{l}
\int_{z=0}^{c} \frac{1}{B\left(1, r^{\prime}-1\right)}\left[1-\frac{\exp \left(\ln \left(z T_{\left(r^{\prime}\right)}+1\right)\right)^{\beta_{1} / \beta_{2}}}{S_{r}}\right]^{r-1}(1-z)^{r^{\prime}-2} d z \\
\text { If }\left(\ln \left(1+S_{(r)}\right)\right)^{1 / \beta_{1}}<\left(\ln \left(1+T_{\left(r^{\prime}\right)}\right)\right)^{1 / \beta_{2}} \\
\int_{z=0}^{1} \frac{1}{B\left(1, r^{\prime}-1\right)}\left[1-\frac{\exp \left(\ln \left(z T_{\left(r^{\prime}\right)}+1\right)\right)^{\beta_{1} / \beta_{2}}}{S_{r}}\right]^{r-1}(1-z)^{r^{\prime}-2} d z \\
\text { If }\left(\ln \left(1+S_{(r)}\right)\right)^{1 / \beta_{1}} \geq\left(\ln \left(1+T_{\left(r^{\prime}\right)}\right)\right)^{1 / \beta_{2}}[16 p t]
\end{array}\right.
$$

where $c=\left[\exp \left(\ln \left(1+S_{(r)}\right)\right)^{\beta_{2} / \beta_{1}}-1\right] / T_{\left(r^{\prime}\right)}$.

Obviously when $\beta_{1}=\beta_{2}$, the UMVUE of $P$ reduces to

$$
\widetilde{P}_{I I}= \begin{cases}\frac{1}{B\left(1, r^{\prime}-1\right)} \sum_{i=0}^{r^{\prime}-2}(-1)^{i}\left(\begin{array}{c}
r^{\prime}-2 \\
i
\end{array}\right)\left(\frac{S_{(r)}}{T_{\left(r^{\prime}\right)}}\right)^{i+1} B(i+1, r) ; & S_{(r)}<T_{\left(r^{\prime}\right)} \\
\frac{1}{B\left(1, r^{\prime}-1\right)} \sum_{j=0}^{r-1}(-1)^{j}\left(\begin{array}{c}
r-1 \\
j
\end{array}\right)\left(\frac{T_{\left(r^{\prime}\right)}}{S_{(r)}}\right)^{j} B\left(j+1, r^{\prime}-1\right) ; & S_{(r)} \geq T_{\left(r^{\prime}\right)}\end{cases}
$$

Using (3), we can write the UMVUE of $h(t)$ at a specified point $t$ as

$$
\widetilde{h}(t)_{I I}=\widetilde{\lambda} e^{t^{\beta}} \beta t^{\beta-1}
$$

Substituting the UMVUE of $\lambda$ in above equation, we obtain

$$
\widetilde{h}(t)_{I I}=\frac{r-1}{S_{(r)}} e^{t^{\beta}} \beta t^{\beta-1}
$$

Furthermore, it can be easily seen from (A.2) (see proof of Lemma 1 in appendix) that, the MLE of $\lambda$ based on Type II censoring is

$$
\widehat{\lambda}_{I I}^{q}=\left(\frac{r}{S_{(r)}}\right)^{q} .
$$

From (1) and one-to-one property of MLE's, the MLE of $f(x)$ is given by

$$
\widehat{f}(x)_{I I}=\widehat{\lambda} \beta e^{x^{\beta}} x^{\beta-1} e^{\widehat{\lambda}\left(1-e^{x^{\beta}}\right)} .
$$

Thus the MLE of $f(x ; \lambda, \beta)$ at a specified point $x$ is given by

$$
\widehat{f}(x)_{I I}=\frac{r}{S_{(r)}} e^{x^{\beta}} \beta x^{\beta-1} \exp \left\{\frac{r}{S_{(r)}}\left(1-e^{x^{\beta}}\right)\right\} .
$$


Using (19), the MLE of $R(t)$ is given by

$$
\begin{aligned}
\widehat{R}(t)_{I I} & =\int_{x=t}^{\infty} \widehat{f}(x ; \lambda, \beta)_{I I} d x \\
& =\int_{x=t}^{\infty} \frac{r}{S_{(r)}} e^{x^{\beta}} \beta x^{\beta-1} \exp \left\{\frac{r}{S_{(r)}}\left(1-e^{x^{\beta}}\right)\right\} d x
\end{aligned}
$$

which on substituting $\frac{r}{S_{(r)}}\left(1-e^{x^{\beta}}\right)=y$ can be written as

$$
\widehat{R}(t)_{I I}=\exp \left\{\frac{-r}{S_{(r)}}\left(e^{t^{\beta}}-1\right)\right\} .
$$

Now to obtain the MLE of $P$, we proceed as follows:

We have

$$
\begin{aligned}
\widehat{P}_{I I} & =\int_{y=0}^{\infty} \int_{x=y}^{\infty} \widehat{f_{I I}}\left(x ; \lambda_{1}, \beta_{1}\right) \widehat{f}_{I I}\left(y ; \lambda_{2}, \beta_{2}\right) d x d y \\
& =\int_{y=0}^{\infty} \widehat{R}\left(y ; \lambda_{1}, \beta_{1}\right)_{I I} \widehat{f}_{I I}\left(y ; \lambda_{2}, \beta_{2}\right) d y
\end{aligned}
$$

which on using (19) and (20) gives

$$
\widehat{P}_{I I}=\int_{y=0}^{\infty} \exp \left\{\frac{-r}{S_{(r)}}\left(e^{y^{\beta_{1}}}-1\right)\right\} \frac{r^{\prime}}{T_{\left(r^{\prime}\right)}} e^{y^{\beta_{2}}} \beta_{2} y^{\beta_{2}-1} \exp \left\{\frac{-r^{\prime}}{T_{\left(r^{\prime}\right)}}\left(e^{y^{\beta_{2}}}-1\right)\right\} d y
$$

Substituting $r^{\prime}\left(e^{y^{\beta_{2}}}-1\right) / T_{\left(r^{\prime}\right)}=z$ in the above expression, we obtain the MLE of $P$ as

$$
\widehat{P}_{I I}=\int_{z=0}^{\infty} \exp \left[\frac{r}{S_{(r)}}\left\{1-\exp \left(\ln \left(\frac{z T_{\left(r^{\prime}\right)}}{r^{\prime}}+1\right)\right)^{\beta_{1} / \beta_{2}}\right\}\right] e^{-z} d z
$$

Moreover, the MLE of $P$ when $\beta_{1}=\beta_{2}$ is given by

$$
\widehat{P}_{I I}=\frac{r^{\prime} S_{(r)}}{r^{\prime} S_{(r)}+r T_{\left(r^{\prime}\right)}}
$$

Using (3) the MLE of $h(t)$ is given by

$$
\widehat{h(t)}_{I I}=\widehat{\lambda} e^{t^{\beta}} \beta t^{\beta-1}
$$

and using (18), we obtain the MLE of $h(t)$ at a specified point $t$ as

$$
\widehat{h(t)}_{I I}=\frac{r}{S_{(r)}} e^{t^{\beta}} \beta t^{\beta-1}
$$

\subsection{Exact Confidence Intervals for $\lambda, R(t)$ and $P$}

Now we consider the problem of constructing two-sided confidence interval for $\lambda$ ( $\beta$ known). The confidence interval is obtained by using pivotal quantity $2 \lambda S_{(r)}$. If we define $\chi^{2}(\nu)$ as the value of $\chi^{2}$ such that

$$
P\left(\chi^{2}>\chi^{2}(\nu)\right)=\int_{\chi^{2}(\nu)}^{\infty} P\left(\chi^{2}\right) d \chi^{2}=\nu
$$


where, $P\left(\chi^{2}\right)$ is the pdf of $\chi^{2}$ distribution with $2 r$ degrees of freedom, then by using the fact that $2 \lambda S_{(r)} \sim \chi_{2 r}^{2}$, the confidence interval is given by

$$
P\left(\frac{\chi^{2}\left(1-\frac{\nu}{2}\right)}{2 S_{(r)}} \leq \lambda \leq \frac{\chi^{2}\left(\frac{\nu}{2}\right)}{2 S_{(r)}}\right)=1-\nu
$$

where $\chi^{2}\left(\frac{\nu}{2}\right)$ and $\chi^{2}\left(1-\frac{\nu}{2}\right)$ are obtained by using (24). Thus for known $\beta, 100(1-\nu) \%$ confidence interval for $\lambda$ is given by

$$
\left(\frac{\chi^{2}\left(1-\frac{\nu}{2}\right)}{2 S_{(r)}}, \frac{\chi^{2}\left(\frac{\nu}{2}\right)}{2 S_{(r)}}\right)
$$

The problem of obtaining the confidence interval for the reliability function $R(t)=\exp \left\{-\lambda\left(e^{t^{\beta}}-1\right)\right\}$ can be solved by noting that $R\left(t_{\circ} ; \lambda\right)$ is a decreasing function of $\lambda$. Thus $\Psi_{1}\left(x_{1}, x_{2}, \ldots, x_{n}\right) \leq \exp \left\{-\lambda\left(e^{t^{\beta}}-1\right)\right\}$ is equivalent to $\lambda \leq \Psi_{1}\left(x_{1}, x_{2}, \ldots, x_{n}\right) / 1-e^{t^{\beta}}$ and $\Psi_{2}\left(x_{1}, x_{2}, \ldots, x_{n}\right) \geq \exp \left\{-\lambda\left(e^{t^{\beta}}-1\right)\right\}$ is equivalent to $\ln \lambda \geq \Psi_{2}\left(x_{1}, x_{2}, \ldots, x_{n}\right) / 1-e^{t^{\beta}}$.

Therefore, the expression

$$
P\left(\Psi_{1}\left(x_{1}, x_{2}, \ldots, x_{n}\right) \leq \exp \left\{-\lambda\left(e^{t_{\circ}^{\beta}}-1\right)\right\} \leq \Psi_{2}\left(x_{1}, x_{2}, \ldots, x_{n}\right)\right)=1-\delta
$$

is equivalent to

$$
P\left(\frac{\ln \Psi_{2}\left(x_{1}, x_{2}, \ldots, x_{n}\right)}{1-e^{t_{\circ}^{\beta}}} \leq \lambda \leq \frac{\ln \Psi_{1}\left(x_{1}, x_{2}, \ldots, x_{n}\right)}{1-e^{t_{\circ}^{\beta}}}\right)=1-\delta
$$

Comparing (25) and (26), it immediately follows that

$$
\chi^{2}\left(1-\frac{\delta}{2}\right) / 2 S_{(r)}=\ln \Psi_{2}\left(x_{1}, x_{2}, \ldots, x_{n}\right) / 1-e^{t^{\beta}}
$$

and

$$
\chi^{2}\left(\frac{\delta}{2}\right) / 2 S_{(r)}=\ln \Psi_{1}\left(x_{1}, x_{2}, \ldots, x_{n}\right) / 1-e^{t_{\circ}^{\beta}}
$$

Therefore

$$
\Psi_{1}=\exp \left[\frac{\left(1-e^{t_{\circ}^{\beta}}\right) \chi^{2}\left(\frac{\delta}{2}\right)}{2 S_{(r)}}\right] \text { and } \Psi_{2}=\exp \left[\frac{\left(1-e^{t_{\circ}^{\beta}}\right) \chi^{2}\left(1-\frac{\delta}{2}\right)}{2 S_{(r)}}\right] .
$$

Thus, $(1-\nu) 100 \%$ confidence interval for $R\left(t_{\circ}, \lambda\right)$ is given by

$$
\left(\exp \left[\frac{\left(1-e^{t_{\circ}^{\beta}}\right) \chi^{2}\left(\frac{\delta}{2}\right)}{2 S_{(r)}}\right], \exp \left[\frac{\left(1-e^{t_{\circ}^{\beta}}\right) \chi^{2}\left(1-\frac{\delta}{2}\right)}{2 S_{(r)}}\right]\right)
$$

In order to obtain the confidence interval for $P$, we utilize the fact that

$$
\frac{2 \lambda_{1} S_{(r)} / 2 r}{2 \lambda_{2} T_{\left(r^{\prime}\right)} / 2 r^{\prime}} \sim F_{2 r, 2 r^{\prime}}
$$

Thus, the confidence interval for $P$ is given by

$$
P\left(F\left(1-\frac{\nu}{2}\right) \leq F \leq F\left(\frac{\nu}{2}\right)\right)=1-\nu
$$


which can further be written as

$$
P\left[\left(\frac{r T_{\left(r^{\prime}\right)} F\left(\frac{\nu}{2}\right)}{r^{\prime} S_{(r)}}+1\right)^{-1} \leq \frac{\lambda_{2}}{\lambda_{1}+\lambda_{2}} \leq\left(\frac{r T_{\left(r^{\prime}\right)} F\left(1-\frac{\nu}{2}\right)}{r^{\prime} S_{(r)}}+1\right)^{-1}\right]=1-\nu
$$

Therefore, $(1-\nu) 100 \%$ confidence interval for $P$ is given by

$$
\left[\left(\frac{r T_{\left(r^{\prime}\right)} F\left(\frac{\nu}{2}\right)}{r^{\prime} S_{(r)}}+1\right)^{-1},\left(\frac{r T_{\left(r^{\prime}\right)} F\left(1-\frac{\nu}{2}\right)}{r^{\prime} S_{(r)}}+1\right)^{-1}\right]
$$

\subsection{Hypothesis Testing}

Under this section, hypothesis testing of the following three cases are considered:

1. Testing of $H_{\circ}: \lambda=\lambda_{\circ}$ against $H_{1}: \lambda \neq \lambda_{\circ}$, when $\beta$ is known

2. Testing of $H_{\circ}: \lambda \leq \lambda_{\circ}$ against $H_{1}: \lambda>\lambda_{\circ}$, when $\beta$ is known

3. Testing of $H_{\circ}: P=P_{\circ}$ against $H_{1}: P \neq P_{\circ}$ when $\beta_{1}=\beta_{2}=\beta$ is known

An important hypothesis in life-testing experiments is $H_{\circ}: \lambda=\lambda_{\circ}$ against $H_{1}: \lambda \neq \lambda_{\circ}$. It follows from (A.2), that the likelihood function for observing $\lambda$ is given by

$$
L(\lambda ; \underline{x}, \beta)=\frac{n !}{(n-r) !} \lambda^{r} \beta^{r} e^{\sum_{i=1}^{r} x_{(i)}^{\beta-1}} \prod_{i=1}^{r} x_{(i)}^{\beta-1} e^{-\lambda S_{(r)}}
$$

Now,

$$
\begin{gathered}
\sup _{\Theta_{\circ}} L(\lambda ; \underline{x}, \beta)=\frac{n !}{(n-r) !} \lambda_{\circ}^{r} \beta^{r} e^{\sum_{i=1}^{r} x_{(i)}^{\beta-1}} \prod_{i=1}^{r} x_{(i)}^{\beta-1} e^{-\lambda_{\circ} S_{(r)}} ; \Theta_{\circ}=\left\{\lambda: \lambda=\lambda_{\circ}\right\}, \\
\sup _{\Theta} L(\lambda ; \underline{x}, \beta)=\frac{n !}{(n-r) !}\left(\frac{r}{S_{(r)}}\right)^{r} \exp \{-r\}
\end{gathered}
$$

Therefore, the likelihood ratio is given by

$$
\begin{aligned}
\Phi(\underline{x}) & =\sup _{\Theta \circ} L(\lambda ; \underline{x}, \beta) / \sup _{\Theta} L(\lambda ; \underline{x}, \beta) \\
& =\left(\frac{\lambda_{\circ} S_{(r)}}{r}\right)^{r} e^{-\left(r+\lambda_{\circ} S_{(r)}\right)}
\end{aligned}
$$

We note that the first term on the right hand side of (32) is an increasing function of $S_{(r)}$ and the second term is monotonically decreasing in $S_{(r)}$. Denoting by $\chi_{2 r}^{2}$, the Chi-square statistics with $2 r$ degrees of freedom and using the fact that $2 \lambda_{\circ} S_{(r)} \sim \chi_{2 r}^{2}$, the critical region is given by

$$
\left\{0<S_{(r)}<k_{\circ}\right\} \cup\left\{k_{\circ}^{\prime}<S_{(r)}<\infty\right\},
$$

where $k_{\circ}$ and $k_{\circ}^{\prime}$ are obtained such that $P\left[\chi_{2 r}^{2}<2 \lambda_{\circ} k_{\circ} \quad\right.$ or $\left.\quad 2 \lambda_{\circ} k_{\circ}^{\prime}<\chi_{2 r}^{2}\right]=\nu$.

Thus,

$$
k_{\circ}=\frac{1}{2 \lambda_{\circ}} \chi_{2 r}^{2}\left(1-\frac{\nu}{2}\right) \quad \text { and } \quad k_{\circ}^{\prime}=\frac{1}{2 \lambda_{\circ}} \chi_{2 r}^{2}\left(\frac{\nu}{2}\right) \text {. }
$$

Another important hypothesis in life testing experiments is $H_{\circ}: \lambda \leq \lambda_{\circ}$ against $H_{1}: \lambda>\lambda_{\circ}$. It follows from 
(29) that, for $\lambda_{1}<\lambda_{2}$,

$$
\frac{h\left(x_{(1)}, x_{(2)}, \ldots, x_{(r)}, \beta, \lambda_{2}\right)}{h\left(x_{(1)}, x_{(2)}, \ldots, x_{(r)}, \beta, \lambda_{1}\right)}=\left(\frac{\lambda_{2}}{\lambda_{1}}\right)^{r} \exp \left(-\left(\lambda_{2}-\lambda_{1}\right) S_{(r)}\right)
$$

It follows from (33) that $h\left(x_{(1)}, x_{(2)}, \ldots, x_{(r)}, \lambda, \beta\right)$ has MLR in $S_{r}$. Thus, the uniformly most powerful critical region (UMPCR) for testing $H_{\circ}: \lambda \leq \lambda_{\circ}$ against $H_{1}: \lambda>\lambda_{\circ}$ (see [15], pp. 88) is given by

$$
\phi\left(x_{(1)}, x_{(2)}, \ldots, x_{(r)}\right)=\left\{\begin{array}{l}
1, S_{r} \leq k_{\circ}^{\prime \prime} \\
0, \text { otherwise }
\end{array}\right.
$$

where $k_{\circ}^{\prime \prime}$ is obtained such that $P\left[\chi_{2 r}^{2}<2 \lambda_{\circ} k_{\circ}^{\prime \prime}\right]=\nu$.

Therefore,

$$
k_{\circ}^{\prime \prime}=\frac{1}{2 \lambda_{\circ}} \chi_{2 r}^{2}(1-\nu) .
$$

Suppose, we want to test $H_{\circ}: P=P_{\circ}$ against $H_{1}: P \neq P_{\circ}$ based on Type II censoring. It follows that $H_{\circ}$ is equivalent to $\lambda_{1}=k \lambda_{2}$. It can be shown that, under $H_{\circ}$

$$
\begin{aligned}
& \widehat{\lambda_{1}}=\frac{k\left(r+r^{\prime}\right)}{k S_{(r)}+T_{\left(r^{\prime}\right)}}, \\
& \widehat{\lambda_{2}}=\frac{r+r^{\prime}}{k S_{(r)}+T_{\left(r^{\prime}\right)}}
\end{aligned}
$$

For a generic constant $K$, the likelihood of observing $\lambda_{1}$ and $\lambda_{2}$, based on $x_{(1)}, x_{(2)}, \ldots, x_{(r)}$ and $y_{(1)}, y_{(2)}, \ldots, y_{\left(r^{\prime}\right)}$ is given by

$$
L\left(\lambda_{1}, \lambda_{2} \mid x_{(1)}, x_{(2)}, \ldots, x_{(r)}, y_{(1)}, y_{(2)}, \ldots, y_{\left(r^{\prime}\right)}\right)=K \lambda_{1}^{r} \lambda_{2}^{r^{\prime}} \exp \left[-\left(\lambda_{1} S_{(r)}+\lambda_{2} T_{\left(r^{\prime}\right)}\right)\right]
$$

Thus,

$$
\begin{gathered}
\sup L\left(\lambda_{1}, \lambda_{2} \mid x_{(1)}, x_{(2)}, \ldots, x_{(r)}, y_{(1)}, y_{(2)}, \ldots, y_{\left(r^{\prime}\right)}\right)=\frac{K k^{r} e^{-\left(r+r^{\prime}\right)}}{\left(k S_{(r)}+T_{\left(r^{\prime}\right)}\right)^{\left(r+r^{\prime}\right)}}, \\
\quad \sup L\left(\lambda_{1}, \lambda_{2} \mid x_{(1)}, x_{(2)}, \underset{\Theta}{,}, x_{(r)}, y_{(1)}, y_{(2)}, \ldots, y_{(s)}\right)=\frac{K e^{-\left(r+r^{\prime}\right)}}{S_{(r)}^{r} T_{\left(r^{\prime}\right)}^{r^{\prime}}} .
\end{gathered}
$$

From (35) and (36), the likelihood ratio criterion is

$$
\lambda^{*}\left(\lambda_{1}, \lambda_{2} \mid x_{(1)}, x_{(2)}, \ldots, x_{(r)}, y_{(1)}, y_{(2)}, \ldots, y_{\left(r^{\prime}\right)}\right)=K \frac{\left(\frac{k S_{(r)}}{T_{\left(r^{\prime}\right)}}\right)^{r}}{\left[1+\frac{k S_{(r)}}{T_{\left(r^{\prime}\right)}}\right]^{r+r^{\prime}}}
$$

Denoting by $F_{a, b}(\cdot)$, the $F$-statistic with $(a ; b)$ degrees of freedom and using the fact that $\frac{S_{r}}{T_{r^{\prime}}} \sim \frac{r \lambda_{2}}{r^{\prime} \lambda_{1}} F_{2 r, 2 r^{\prime}}(\cdot)$, the critical region is given by

$$
\left\{\frac{S_{(r)}}{T_{\left(r^{\prime}\right)}}<k_{2} \quad \text { and } \quad \frac{S_{(r)}}{T_{\left(r^{\prime}\right)}}>k_{2}^{\prime}\right\},
$$

where $k_{2}$ and $k_{2}^{\prime}$ are obtained such that

$$
P\left\{\frac{r^{\prime} k S_{(r)}}{r T_{\left(r^{\prime}\right)}}<F_{2 r, 2 r^{\prime}} \cup \frac{r^{\prime} k S_{(r)}}{r T_{\left(r^{\prime}\right)}}>F_{2 r, 2 r^{\prime}}\right\}=\nu .
$$

Thus,

$$
k_{2}=\frac{r}{r^{\prime} k} F_{2 r, 2 r^{\prime}}\left(1-\frac{\nu}{2}\right) \quad \text { and } \quad k_{2}^{\prime}=\frac{r}{r^{\prime} k} F_{2 r, 2 r^{\prime}}\left(\frac{\nu}{2}\right) .
$$




\section{Estimation and Testing Procedures Based on the Sampling Scheme of Bartholomew}

Throughout this section, we assume that $n$ items are put on a test and we terminate the life testing experiment at a preassigned time $t_{0}$. Suppose we carry out time-censored test where the items that fail are immediately replaced. Denoting $X_{(1)} \leq X_{(2)} \leq \ldots \leq X_{(n)}$ be the failure times of $n$ items under a test from (1), the test begins at time $X_{(0)}=0$ and the system operates till $X_{(1)}=x_{1}$, when the first failure occurs. The failed item is replaced by a new one and the system operates till the second failure occurs at time $X_{(2)}=x_{2}$ and so on. The experiment is terminated at time $t_{\circ}$. Here, $X_{(i)}$ is the time until $i^{\text {th }}$ failure measured from time 0 .

Now we provide an important lemma, which will be useful in deducing the main results of this section.

Lemma 2

If $N\left(t_{\circ}\right)$ be the number of failures during the interval $\left[0, t_{\circ}\right]$, then

$$
P\left[N\left(t_{\circ}\right)=r \mid t_{\circ}\right]=\frac{\exp \left\{-n \lambda\left(e^{t_{\circ}^{\beta}}-1\right)\right\}\left\{n \lambda\left(e^{t_{\circ}^{\beta}}-1\right)\right\}^{r}}{r !} .
$$

Proof The proof of Lemma 2 can be found in the Appendix.

\subsection{UMVUE's and MLE's of $\lambda^{q}, R(t), P$ and $h(t)$}

It follows from Lemma 2 and Fisher-Neyman factorization theorem (see [19], pp. 347) that $N\left(t_{\circ}\right)$ is sufficient for $\lambda$. Moreover, since the distribution of $N\left(t_{\circ}\right)$ belongs to exponential family, it is also complete. Thus the $q^{\text {th }}$ factorial moment of the distribution of $N\left(t_{\circ}\right)$ is given by

$$
E\left[N\left(t_{\circ}\right)\left(N\left(t_{\circ}\right)-1\right)\left(N\left(t_{\circ}\right)-2\right) \ldots\left(N\left(t_{\circ}\right)-q+1\right)\right]=\left[n \lambda\left(e^{t_{\circ}^{\beta}}-1\right)\right]^{q} .
$$

Hence for any positive integer $q$ and $N\left(t_{\circ}\right)=r$, the UMVUE of $\lambda^{q}$ is given by

$$
\widetilde{\lambda}_{I}^{q}= \begin{cases}\frac{r !}{(r-q) !}\left[n\left(e^{t_{\circ}^{\beta}}-1\right)\right]^{-q} & : r-q>0 \\ 0 & : \text { otherwise }\end{cases}
$$

Further, if we write the pdf (1) as,

$$
\begin{aligned}
f(x ; \lambda, \beta) & =\lambda \beta e^{x^{\beta}} x^{\beta-1} e^{\lambda\left(1-e^{x^{\beta}}\right)}, \\
& =\lambda \beta e^{x^{\beta}} x^{\beta-1} \sum_{i=0}^{\infty} \frac{(-1)^{i}}{i !}\left(e^{x^{\beta}}-1\right)^{i} \lambda^{i} .
\end{aligned}
$$

then with the help of (39), the UMVUE of $f(x ; \lambda, \beta)$ at a specified point $x$ is found to be

$$
\widetilde{f}_{I}(x ; \lambda, \beta)= \begin{cases}\frac{r \beta e^{x^{\beta}} x^{\beta-1}}{n\left(e^{t_{\circ}^{\beta}}-1\right)}\left(1-\frac{e^{x^{\beta}}-1}{n\left(e^{t_{\circ}^{\beta}}-1\right)}\right)^{r-1} & : e^{x^{\beta}}-1<n\left(e^{t_{\circ}^{\beta}}-1\right) \\ 0 & : \text { otherwise }\end{cases}
$$

Using (40), the UMVUE of $R(t)$ is given by

$$
\begin{aligned}
\widetilde{R}(t)_{I} & =\int_{t}^{\infty} \widetilde{f}_{I}(x ; \lambda, \beta) d x \\
& =\int_{t}^{\infty} \frac{r \beta e^{x^{\beta}} x^{\beta-1}}{n\left(e^{t^{\beta}}-1\right)}\left(1-\frac{e^{x^{\beta}}-1}{n\left(e^{t_{\circ}^{\beta}}-1\right)}\right)^{r-1} d x .
\end{aligned}
$$


Substituting $\frac{e^{x^{\beta}}-1}{n\left(e^{t_{\circ}^{\beta}}-1\right)}=z$ in the above expression, we obtain the UMVUE of $R(t)$ as

$$
\widetilde{R}(t)_{I}= \begin{cases}{\left[1-\frac{e^{t^{\beta}}-1}{n\left(e^{t_{\circ}^{\beta}}-1\right)}\right]^{r}} & : e^{t^{\beta}}-1<n\left(e^{t_{\circ}^{\beta}}-1\right) \\ 0 & : \text { otherwise }\end{cases}
$$

Let $n$ items on $X$ and $m$ on $Y$ be put on a life test, where $X$ and $Y$ follow distribution with pdf (8) and (9) respectively. Let $t_{\circ}$ and $r$ be the termination times and number of failures before the termination time for $X$ and $t_{\circ \circ}$ and $r^{\prime}$ be the corresponding figures for $Y$. Obviously, using (40), the UMVUE's of $f_{1}\left(x ; \lambda_{1}, \beta_{1}\right)$ and $f_{2}\left(y ; \lambda_{2}, \beta_{2}\right)$ based on the sampling scheme of Bartholomew are given by

$$
\begin{aligned}
& \widetilde{f}_{1 I}\left(x ; \lambda_{1}, \beta_{1}\right)=\frac{r \beta_{1} e^{x^{\beta_{1}}} x^{\beta_{1}-1}}{n\left(e^{t_{\circ}^{\beta_{1}}}-1\right)}\left(1-\frac{e^{x^{\beta_{1}}}-1}{n\left(e^{t_{\circ}^{\beta_{1}}}-1\right)}\right)^{r-1} ; e^{x^{\beta_{1}}}-1<n\left(e^{t_{\circ}^{\beta_{1}}}-1\right), \\
& \widetilde{f}_{2 I}\left(y ; \lambda_{2}, \beta_{2}\right)=\frac{r^{\prime} \beta_{2} e^{y^{\beta_{2}}} y^{\beta_{2}-1}}{m\left(e^{t_{\circ}^{\beta_{2}}}-1\right)}\left(1-\frac{e^{y^{\beta_{2}}}-1}{m\left(e^{t_{\circ}^{\beta_{2}}}-1\right)}\right)^{r^{\prime}-1} ; e^{y^{\beta_{2}}}-1<m\left(e^{t_{\circ}^{\beta_{2}}}-1\right) .
\end{aligned}
$$

Then the UMVUE of $P$ is obtained by solving

$$
\begin{aligned}
\widetilde{P}_{I} & =\int_{y=0}^{\infty} \int_{x=y}^{\infty} \widetilde{f}_{1 I}\left(x ; \lambda_{1}, \beta_{1}\right) \widetilde{f}_{2 I}\left(y ; \lambda_{2}, \beta_{2}\right) d x d y \\
& =\int_{y=0}^{\infty} \widetilde{R}_{I}\left(y ; \lambda_{1}, \beta_{1}\right) \widetilde{f}_{2 I}\left(y ; \lambda_{2}, \beta_{2}\right) d y
\end{aligned}
$$

which on using (41) and (43) leads to

$$
\begin{aligned}
& \widetilde{P}_{I}=\int_{y=0}^{\infty}\left[1-\frac{e^{y^{\beta_{1}}}-1}{n\left(e^{t_{\circ}^{\beta_{1}}}-1\right)}\right]^{r} \frac{r^{\prime} \beta_{2} e^{y^{\beta_{2}}} y^{\beta_{2}-1}}{m\left(e^{t_{\circ}^{\beta_{2}}}-1\right)}\left(1-\frac{e^{y^{\beta_{2}}}-1}{m\left(e^{t_{\circ} \beta_{2}}-1\right)}\right)^{r^{\prime}-1} d y \\
& e^{y^{\beta_{1}}}-1<n\left(e^{t_{\circ}^{\beta_{1}}}-1\right), \quad e^{y^{\beta_{2}}}-1<m\left(e^{t_{\circ \circ}^{\beta_{2}}}-1\right)
\end{aligned}
$$

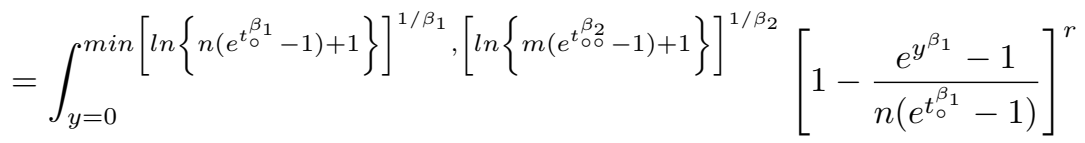

$$
\begin{aligned}
& \times \frac{r^{\prime} \beta_{2} e^{y^{\beta_{2}}} y^{\beta_{2}-1}}{m\left(e^{t_{\circ} \circ}-1\right)}\left(1-\frac{e^{y^{\beta_{2}}}-1}{m\left(e^{t_{\circ}^{\beta_{2}}}-1\right)}\right)^{r^{\prime}-1} d y
\end{aligned}
$$


Considering (45) and substituting $\frac{e^{y^{\beta_{2}}}-1}{m\left(e^{t_{\circ}^{\beta_{2}}}-1\right)}=z$, we obtain the UMVUE of $P$ as

$$
\widetilde{P}_{I}=\left\{\begin{array}{l}
\int_{z=0}^{c}\left[1-\frac{\exp \left\{\ln \left(m\left(e^{t_{\circ} \circ}-1\right) z+1\right)\right\}^{\beta_{2} / \beta_{1}}-1}{n\left(e^{t_{\circ}}-1\right)}\right]^{r} r^{\prime}(1-z)^{r^{\prime}-1} d z \\
{\left[\ln \left\{n\left(e^{t_{\circ}^{\beta_{1}}}-1\right)+1\right\}\right]^{1 / \beta_{1}} \leq\left[\ln \left\{m\left(e^{t_{\circ}^{\beta_{2}}}-1\right)+1\right\}\right]^{1 / \beta_{2}}} \\
\int_{z=0}^{1}\left[1-\frac{\exp \left\{\ln \left(m\left(e^{t_{\circ \circ}}-1\right) z+1\right)\right\}^{\beta_{2} / \beta_{1}}-1}{n\left(e^{t_{\circ}}-1\right)}\right]^{r} r^{\prime}(1-z)^{r^{\prime}-1} d z \\
{\left[\ln \left\{n\left(e^{t_{\circ}^{\beta_{1}}}-1\right)+1\right\}\right]^{1 / \beta_{1}}>\left[\ln \left\{m\left(e^{t_{\circ}^{\beta_{2}}}-1\right)+1\right\}\right]^{1 / \beta_{2}}}
\end{array}\right.
$$

where $c=\left(\exp \left[\ln \left\{n\left(e^{t_{\circ}^{\beta_{1}}}-1\right)+1\right\}\right]^{\beta_{2} / \beta_{1}}-1\right) /\left(m\left(e^{t_{\circ \circ}^{\beta_{2}}}-1\right)\right)$.

As a special case if $\beta_{1}=\beta_{2}$, the UMVUE of $P$ is given by

$$
\widetilde{P_{I}}= \begin{cases}s \sum_{i=0}^{s-1}(-1)^{i}\left(\begin{array}{c}
r^{\prime}-1 \\
i
\end{array}\right)\left(\frac{n}{m}\right)^{i+1} B(i+1, r+1) & : n \leq m \\
s \sum_{j=0}^{r}(-1)^{j}\left(\begin{array}{c}
r \\
j
\end{array}\right)\left(\frac{m}{n}\right)^{j} B\left(j+1, r^{\prime}\right) & : n>m\end{cases}
$$

Further, using (3), the UMVUE of $h(t)$ at a specified point $t$ is given by

$$
\widetilde{h}(t)_{I}=\widetilde{\lambda} e^{t^{\beta}} \beta t^{\beta-1}
$$

which on using (39) gives that

$$
\widetilde{h(t)}{ }_{I}=\frac{r}{n\left(e^{t^{\beta}}-1\right)} e^{t^{\beta}} \beta t^{\beta-1} .
$$

Moreover, it can be easily seen from Lemma 2 that the MLE of $\lambda^{q}$ based on the sampling scheme of Bartholomew is given by

$$
\widehat{\lambda}_{I}^{q}=\left(\frac{r}{n\left(e^{t_{o}^{\beta}}-1\right)}\right)^{q}
$$

Using (2), the expression of $R(t)$ at a point $t$ is given by

$$
R(t)=\exp \left\{\lambda\left(1-e^{t^{\beta}}\right)\right\}
$$

Now from (49)-(50) and one-to-one property of MLEs, we obtain the MLE of $R(t)$, based on the sampling scheme of Bartholomew as

$$
\widehat{R}(t)_{I}=\exp \left\{\frac{r\left(1-e^{t^{\beta}}\right)}{n\left(e^{t_{\circ}^{\beta}}-1\right)}\right\}
$$

Further the MLE of $f(x ; \lambda, \beta)$ at a specified point $x$ is

$$
\widehat{f}_{I}(x ; \lambda, \beta)=\exp \left\{\frac{r\left(1-e^{x^{\beta}}\right)}{n\left(e^{t_{\circ}^{\beta}}-1\right)}\right\} \frac{r \beta e^{x^{\beta}} x^{\beta-1}}{n\left(e^{t_{\circ}^{\beta}}-1\right)}
$$


To obtain the MLE of $P$, we proceed as follows:

The expression of the MLE of $P$ is given by

$$
\begin{aligned}
\widehat{P}_{I} & =\int_{y=0}^{\infty} \int_{x=y}^{\infty} \widehat{f}_{I}\left(x ; \lambda_{1}, \beta_{1}\right) \widehat{f}_{I}\left(y ; \lambda_{2}, \beta_{2}\right) d x d y \\
& =\int_{y=0}^{\infty} \widehat{R}_{I}\left(y ; \lambda_{1}, \beta_{1}\right) \widehat{f}_{I}\left(y ; \lambda_{2}, \beta_{2}\right) d y
\end{aligned}
$$

which on using (51) and (52) gives that

$$
\widehat{P}=\int_{y=0}^{\infty} \exp \left\{\frac{r\left(1-e^{y^{\beta_{1}}}\right)}{n\left(e^{\beta_{\circ}^{\beta_{1}}}-1\right)}\right\} \exp \left\{\frac{r^{\prime}\left(1-e^{y^{\beta_{2}}}\right)}{m\left(e^{t_{\circ}^{\beta_{2}}}-1\right)}\right\} \frac{r^{\prime} \beta_{2} e^{y^{\beta_{2}}} y^{\beta_{2}-1}}{m\left(e^{t_{\circ}^{\beta_{2}}}-1\right)} d y
$$

Substituting $\left[r^{\prime}\left(e^{y^{\beta_{2}}}-1\right] /\left[m\left(e^{t_{\circ}^{\beta_{2}}}-1\right)\right]=z\right.$ in above expression, we obtain the MLE of $P$ as:

$$
\widehat{P}_{I}=\int_{z=0}^{\infty} \exp \left[\frac{-r\left\{\exp \left(\ln \left(\frac{m\left(e^{t_{\circ}^{\beta_{0}}}-1\right) z+1}{s}\right)+1\right)^{\beta_{1} / \beta_{2}}-1\right\}}{n\left(e^{t_{\circ}^{\beta_{1}}}-1\right)}\right] e^{-z} d z
$$

Further, the MLE of $P$ when $\beta_{1}=\beta_{2}$ and $t_{\circ}=t_{\circ \circ}$, is given by:

$$
\widehat{P}=\frac{r^{\prime} n}{r^{\prime} n+r m}
$$

Using (3), the MLE of $h(t)$ is given by

$$
\widehat{h}(t)_{I}=\widehat{\lambda} e^{t^{\beta}} \beta t^{\beta-1}
$$

Using (49) and one-to-one property of MLE's, the MLE of $h(t)$, based on the sampling scheme of Bartholomew is now given by

$$
\widehat{h}(t)_{I}=\frac{r}{n\left(e^{t_{\circ}^{\beta}}-1\right)} e^{t^{\beta}} \beta t^{\beta-1}
$$

\subsection{Hypothesis Testing}

Under this section, we consider the hypothesis testing based on the sampling scheme of Bartholomew, for the following two cases:

1. Testing of $H_{\circ}: \lambda=\lambda$. against $H_{1}: \lambda \neq \lambda_{\circ}$, when $\beta$ is known

2. Testing of $H_{\circ}: \lambda \leq \lambda$. against $H_{1}: \lambda>\lambda_{\circ}$, when $\beta$ is known

Proceeding in a similar manner as in Section 2.3 and using Lemma 2, it can be shown that, based on the sampling scheme of Bartholomew, the critical region for testing $H_{\circ}: \lambda=\lambda$. against $H_{1}: \lambda \neq \lambda_{\circ}$ is given by:

$$
\left\{r<k_{1} \quad \text { or } \quad r>k_{1}^{\prime}\right\} \quad \text { where } r \sim \operatorname{Poisson}\left(n \lambda\left(e^{t_{\circ}^{\beta}}-1\right)\right)
$$

On the similar lines as in Section 2.3 and using Lemma 2, it can be shown that, based on the sampling scheme of Bartholomew, the uniformly most powerful critical region for testing $H_{\circ}: \lambda \leq \lambda$. against $H_{1}: \lambda>\lambda_{\circ}$ is given by:

$$
\phi(r)= \begin{cases}1 & ; r \geq K_{1}^{\prime} \\ 0 & ; \text { otherwise }\end{cases}
$$




\section{Simulation Study}

In this section, we study the performance of our estimation and testing procedures through simulations. Throughout this section, comparisons are made on the basis of MSEs of estimators and simulation experiments are conducted using Monte Carlo simulation technique.

\subsection{Simulation based on Estimation Procedures}

First we compare the performance of estimators of $\lambda^{q}, R(t), P$ and $h(t)$ based on Type II censoring scheme. For this purpose, we have generated 1000 random samples from (1) each of size $n=50$ for $(\lambda, \beta)=(0.5,0,5),(0.5,1)$, $(0.5,2),(0.5,4)$. For each sample, we arranged the data in ascending order and considered a sample of first ' $r$ ' $(r \leq n)$ observations.

For different values of $r=10,20,30$ and 50, we have computed average values of $\widetilde{\lambda}_{I I}$ and $\widehat{\lambda}_{I I}$ and their corresponding MSE's and results are reported in Table 1. Similiarly, we obtain average length and coverage probability of interval estimates which are reported in Table 2.

Table 1. Average values of point estimates of $\lambda$ and their MSEs/Variances, when $\beta$ is known

\begin{tabular}{ccccccccc}
\hline$r->$ & \multicolumn{2}{c}{$\mathbf{1 0}$} & \multicolumn{2}{c}{$\mathbf{2 0}$} & \multicolumn{2}{c}{ 30 } & \multicolumn{2}{c}{$\mathbf{5 0}$} \\
$\beta$ & $\tilde{\lambda}$ & $\hat{\lambda}$ & $\tilde{\lambda}$ & $\hat{\lambda}$ & $\tilde{\lambda}$ & $\hat{\lambda}$ & $\tilde{\lambda}$ & $\hat{\lambda}$ \\
\hline \multirow{2}{*}{0.5} & 0.4987 & 0.5541 & 0.4978 & 0.524 & 0.5033 & 0.5207 & 0.4993 & 0.5095 \\
& 0.0265 & 0.0357 & 0.0142 & 0.0163 & 0.0094 & 0.0105 & 0.0052 & 0.0055 \\
\hline \multirow{2}{*}{1} & 0.5065 & 0.5627 & 0.5015 & 0.5279 & 0.5044 & 0.5218 & 0.4992 & 0.5094 \\
& 0.0367 & 0.0492 & 0.0133 & 0.0155 & 0.0092 & 0.0103 & 0.0051 & 0.0054 \\
\hline \multirow{2}{*}{2} & 0.5062 & 0.5624 & 0.5047 & 0.5313 & 0.4968 & 0.5139 & 0.5 & 0.5102 \\
& 0.0333 & 0.045 & 0.0147 & 0.0172 & 0.009 & 0.0099 & 0.0048 & 0.0051 \\
\hline \multirow{2}{*}{4} & 0.4973 & 0.5526 & 0.5 & 0.5263 & 0.5033 & 0.5207 & 0.4981 & 0.5083 \\
& 0.0281 & 0.0375 & 0.0128 & 0.0149 & 0.009 & 0.01 & 0.0053 & 0.0056 \\
\hline
\end{tabular}

Note: 1st and 2nd rows represent the average estimates and MSE's of $\lambda$.

Table 2. Average length and coverage probability of interval estimates

\begin{tabular}{ccccccccc}
\hline$r->$ & \multicolumn{2}{c}{$\mathbf{1 0}$} & \multicolumn{2}{c}{$\mathbf{2 0}$} & \multicolumn{2}{c}{$\mathbf{5 0}$} \\
$\beta$ & $A . L$. & $C . P$. & $A . L$. & C.P. & A.L. & C.P. & A.L. & C.P. \\
\hline 0.5 & 0.6801 & 95.05 & 0.4562 & 95.2 & 0.3706 & 95.75 & 0.2817 & 95.05 \\
\hline 1 & 0.6841 & 95 & 0.4555 & 95.7 & 0.3705 & 95.05 & 0.2825 & 95.7 \\
\hline 2 & 0.6860 & 94.98 & 0.4621 & 95.06 & 0.3692 & 95.16 & 0.2832 & 95.15 \\
\hline 4 & 0.6842 & 94.88 & 0.4575 & 95.32 & 0.3691 & 95.18 & 0.2822 & 95.6 \\
\hline \multicolumn{4}{c}{ Note: A.L.: Average Length, C.P.: Coverage Probability }
\end{tabular}

From Table 1, it is observed that, the MSE corresponding to UMVUE is much lower than the MLE. Thus, we can say that the performance of UMVUE of $\lambda$ based on Type II censoring is much better than MLE. It can also be seen from Table 1 that, as $r$ increases, performance of both the estimators improve (as MSE is decreasing) and estimates come closer to each other. Also from Table 2, we observe that, as the truncation number $r$ increases, the length of confidence intervals decrease. This justifies the fact that as $r$ moves closer to $n$, the precision of our estimate will increase .

Again for $r=10,20,30$ and 50, we have computed the average values of $\widetilde{R(t)}, \widehat{R(t)}$ and their corresponding MSE's and the results are reported in Table 3. Similiarly, we obtain average length and coverage probability of 
interval estimates which are reported in Table 4.

Table 3. Average values of point estimates of $R(t)$ and their MSEs/Variances, when $\beta$ is known

\begin{tabular}{cccccccccc}
\hline \multicolumn{2}{c}{$r->$} & \multicolumn{2}{c}{$\mathbf{1 0}$} & \multicolumn{2}{c}{$\mathbf{2 0}$} & \multicolumn{2}{c}{ 30 } & \multicolumn{2}{c}{$\mathbf{5 0}$} \\
$t \downarrow$ & $R(t) \downarrow$ & $\widetilde{R(t)}$ & $\widetilde{R(t)}$ & $\widetilde{R(t)}$ & $\widetilde{R(t)}$ & $\widetilde{R(t)}$ & $\widehat{R(t)}$ & $\widetilde{R(t)}$ & $\widehat{R(t)}$ \\
\hline \multirow{2}{*}{0.2} & \multirow{2}{*}{0.9798} & 0.9795 & 0.9772 & 0.9798 & 0.97880 & 0.9797 & 0.97899 & 0.9799 & 0.9794 \\
& & $5.3 \mathrm{e}-05$ & $7.1 \mathrm{e}-05$ & $2.1 \mathrm{e}-05$ & $2.4 \mathrm{e}-05$ & $1.5 \mathrm{e}-05$ & $1.7 \mathrm{e}-05$ & $8 \mathrm{e}-06$ & $9 \mathrm{e}-06$ \\
\hline \multirow{2}{*}{0.5} & \multirow{2}{*}{0.8676} & 0.8681 & 0.8558 & 0.8688 & 0.8629 & 0.8683 & 0.8644 & 0.8667 & 0.8644 \\
& & 0.0016 & 0.002 & $8 \mathrm{e}-04$ & $9 \mathrm{e}-04$ & $5 \mathrm{e}-04$ & $5 \mathrm{e}-04$ & $3 \mathrm{e}-04$ & $3 \mathrm{e}-04$ \\
\hline \multirow{2}{*}{0.8} & \multirow{2}{*}{0.6388} & 0.6453 & 0.6231 & 0.639 & 0.6279 & 0.6401 & 0.6327 & 0.64 & 0.6355 \\
& & 0.0089 & 0.0093 & 0.0044 & 0.0045 & 0.0025 & 0.0026 & 0.0016 & 0.0016 \\
\hline \multirow{2}{*}{0.9} & \multirow{2}{*}{0.5358} & 0.5356 & 0.5132 & 0.5361 & 0.524705 & 0.5349 & 0.5272 & 0.5357 & 0.5311 \\
& & 0.0125 & 0.0125 & 0.00575 & 0.0058 & 0.0038 & 0.0038 & 0.0023 & 0.0023 \\
\hline \multirow{2}{*}{1} & \multirow{2}{*}{0.4235} & 0.4246 & 0.4052 & 0.4239 & 0.4138 & 0.4237 & 0.4169 & 0.4241 & 0.4199 \\
& & 0.0141 & 0.0132 & 0.0068 & 0.0067 & 0.0045 & 0.0044 & 0.0027 & 0.0026 \\
\hline \multirow{2}{*}{1.5} & \multirow{2}{*}{0.1998} & 0.201 & 0.196 & 0.2008 & 0.198 & 0.201 & 0.1991 & 0.1996 & 0.1984 \\
& & 0.0108 & 0.0091 & 0.0051 & 0.0047 & 0.0035 & 0.0033 & 0.0021 & 0.002 \\
\hline
\end{tabular}

Note: 1st and 2nd rows represent the average estimates and MSE of $R(t)$.

Table 4. Average length and coverage probability of interval estimates

\begin{tabular}{ccccccccc}
\hline$r->$ & \multicolumn{2}{c}{$\mathbf{1 0}$} & \multicolumn{2}{c}{$\mathbf{3 0}$} & \multicolumn{2}{c}{$\mathbf{5 0}$} \\
$t$ & $A . L$. & $C . P$. & $A . L$. & $C . P$. & A.L. & C.P. & A.L. & C.P. \\
\hline 0.2 & 0.027 & 95 & 0.0183 & 94.8 & 0.0148 & 94.6 & 0.0113 & 95.6 \\
\hline 0.5 & 0.1608 & 95.1 & 0.111 & 95.04 & 0.0895 & 95.02 & 0.0689 & 94.76 \\
\hline 0.8 & 0.3466 & 94.68 & 0.2483 & 095.07 & 0.2034 & 94.59 & 0.1582 & 094.98 \\
\hline 0.9 & 0.4176 & 95 & 0.3068 & 94.98 & 0.2537 & 94.91 & 0.1987 & 95.26 \\
\hline 1 & 0.4176 & 95 & 0.3068 & 94.98 & 0.2537 & 94.91 & 0.1987 & 95.26 \\
\hline 1.5 & 0.1303 & 94.99 & 0.0748 & 95.22 & 0.0559 & 95.05 & 0.0396 & 95.03 \\
\hline \multicolumn{8}{c}{ Note: A.L.: Average Length, C.P.: Coverage Probability }
\end{tabular}

Comparing the estimates on the basis of MSE's, obtained in Table 3, it can be seen that, based on Type II censoring, for different values of $\mathrm{t}$, the performance of UMVUE of $R(t)$ is better than the performance of MLE of $R(t)$. However, for $t=1$ and beyond, the performance of MLE is better than UMVUE. Performance of both the estimators is quite similar in case of large values of $r$. From Table 3, it is also clear that, as $r$ increases, the MSE corresponding to both the estimators decrease. Also from Table 4, we observe that as the truncation number $r$ increases, the length of confidence intervals decreases. It establishes the improvement in estimate of $R(t)$ for increasing values of $r$.

In order to investigate the performance of the estimators of $P$, we have generated 1000 random samples from each of the populations $X$ and $Y$ with sizes $(\mathrm{n}, \mathrm{m})$ from (1.1) with $\beta_{1}=\beta_{2}=2$ and $\left(\lambda_{1}, \lambda_{2}\right)=(0.5,0.5),(0.5,1)$, $(0.5,1.5)$ and $(0.5,2)$. The samples corresponding to both the populations are arranged in ascending order and first $\left(r, r^{\prime}\right)$ observations are considered. For $\left(r, r^{\prime}\right)=(10,10),(20,20),(30,25)$ and $(40,40)$, we have computed average values of $\widetilde{P}$ and $\widehat{P}$ and their corresponding MSE's and the results are presented in Table 5. Similiarly, we obtain 
average length and coverage probability of interval estimates which are reported in Table 6.

Table 5. UMVUE's and MLE's of $P$

\begin{tabular}{|c|c|c|c|c|c|c|c|c|}
\hline \multirow{4}{*}{$\begin{array}{c}\lambda_{1}-> \\
\lambda_{2}-> \\
P-> \\
\left(r, r^{\prime}\right) \downarrow\end{array}$} & \multicolumn{2}{|c|}{0.5} & \multicolumn{2}{|c|}{0.5} & \multicolumn{2}{|c|}{0.5} & \multicolumn{2}{|c|}{0.5} \\
\hline & \multicolumn{2}{|c|}{0.5} & \multicolumn{2}{|c|}{1} & \multicolumn{2}{|c|}{1.5} & \multicolumn{2}{|c|}{2} \\
\hline & \multicolumn{2}{|c|}{0.5} & \multicolumn{2}{|c|}{0.6666667} & \multicolumn{2}{|c|}{0.75} & \multicolumn{2}{|c|}{0.8} \\
\hline & $\widetilde{P}$ & $\widehat{P}$ & $\widetilde{P}$ & $\widehat{P}$ & $\widetilde{P}$ & $\widehat{P}$ & $\widetilde{P}$ & $\widehat{P}$ \\
\hline \multirow{2}{*}{$(10,10)$} & 0.5029 & 0.5028 & 0.6614 & 0.6545 & 0.7461 & 0.737 & 0.8039 & 0.7943 \\
\hline & 0.013 & 0.0118 & 0.0112 & 0.0105 & 0.0084 & 0.0082 & 0.0055 & 0.0055 \\
\hline \multirow{2}{*}{$(20,20)$} & 0.4994 & 0.4994 & 0.6688 & 0.6652 & 0.7506 & 0.7459 & 0.801 & 0.7961 \\
\hline & 0.0063 & 0.006 & 0.0054 & 0.0052 & 0.0034 & 0.0034 & 0.0024 & 0.0024 \\
\hline \multirow{2}{*}{$(30,25)$} & 0.6242 & 0.6229 & 0.6655 & 0.6635 & 0.7502 & 0.7474 & 0.8004 & 0.7971 \\
\hline & 0.0042 & 0.0041 & 0.0036 & 0.0035 & 0.0027 & 0.0026 & 0.0018 & 0.0018 \\
\hline \multirow{2}{*}{$(40,40)$} & 0.4991 & 0.4991 & 0.6666 & 0.6647 & 0.7511 & 0.7488 & 0.7998 & 0.7974 \\
\hline & 0.0032 & 0.0032 & 0.0024 & 0.0024 & 0.0017 & 0.0017 & 0.0014 & 0.0014 \\
\hline
\end{tabular}

Note: 1st and 2nd rows represent the average estimates and MSE's.

Table 6. Average length and coverage probability of interval estimates

\begin{tabular}{|c|c|c|c|c|c|c|c|c|}
\hline$\lambda_{1}->$ & \multicolumn{2}{|c|}{0.5} & \multicolumn{2}{|c|}{0.5} & \multicolumn{2}{|c|}{0.5} & \multicolumn{2}{|c|}{0.5} \\
\hline$\lambda_{2}->$ & \multicolumn{2}{|c|}{0.5} & \multicolumn{2}{|c|}{1} & \multicolumn{2}{|c|}{1.5} & \multicolumn{2}{|c|}{2} \\
\hline$P->$ & \multicolumn{2}{|c|}{0.5} & \multicolumn{2}{|c|}{0.6666667} & \multicolumn{2}{|c|}{0.75} & \multicolumn{2}{|c|}{0.8} \\
\hline$\left(r, r^{\prime}\right) \downarrow$ & A.L. & C.P. & A.L. & C.P. & A.L. & C.P. & A.L. & C.P. \\
\hline$(10,10)$ & 0.4054 & 94.64 & 0.3716 & 94.63 & 0.3267 & 94.96 & 0.2879 & 94.78 \\
\hline$(20,20)$ & 0.2977 & 95.15 & 0.2687 & 94.95 & 0.232 & 95.09 & 0.2015 & 95.08 \\
\hline$(30,25)$ & 0.2575 & 94.75 & 0.232 & 94.76 & 0.1991 & 94.67 & 0.1723 & 95.11 \\
\hline$(40,40)$ & 0.2147 & 94.91 & 0.1927 & 94.82 & 0.1642 & 95.11 & 0.1415 & 95.07 \\
\hline
\end{tabular}

Note: A.L.: Average Length, C.P.: Coverage Probability

From Table 5, it is clear that based on Type II censoring, for all values of $\left(r, r^{\prime}\right)$, MLE of $P$ gives better results than UMVUE of $P$. Also from Table 6 , we observe that, as truncation number $\left(r, r^{\prime}\right)$ increases, the length of confidence intervals decrease. It establishes the improvement in estimate of $P$ for increasing values of $\left(r, r^{\prime}\right)$.

To compare the estimates of $h(t)$, we have plotted the hazard rates and their estimates against time $t$ for $\lambda=2$, $\beta=0.8, n=50$ and $r=10,20,30$ and 40. In Figure 1, we have plotted the hazard rate and it's MLE and UMVUE for different values of $r$.

We observe that, as $r$ increases, the estimates come closer to the true values. For $r=40$, the estimated values of hazard rate overlaps the plot. It establishes the consistency properties of estimators.

Now we compare the performance of estimators of $\lambda^{q}, R(t), P$ and $h(t)$ based on the sampling scheme of Bartholomew.

In order to obtain the point estimates of $R(t)$ based on the sampling scheme of Bartholomew, we have generated 1000 random samples each of size 100 from (1) with $\lambda=0.5$ and $\beta=2$. By fixing the termination time at $t_{0}$, and replacing the failure by operating one, values of $r$ (number of failures before time $t_{\circ}$ ) are computed. For different termination times $t_{\circ}=0.20,0.45,0.50,0.65$ and 0.80 , we have computed average values of $\widehat{R(t)}$ and $\widehat{R(t)}$, their corresponding MSE's. For different values of $t$, results are presented in Table 7. 


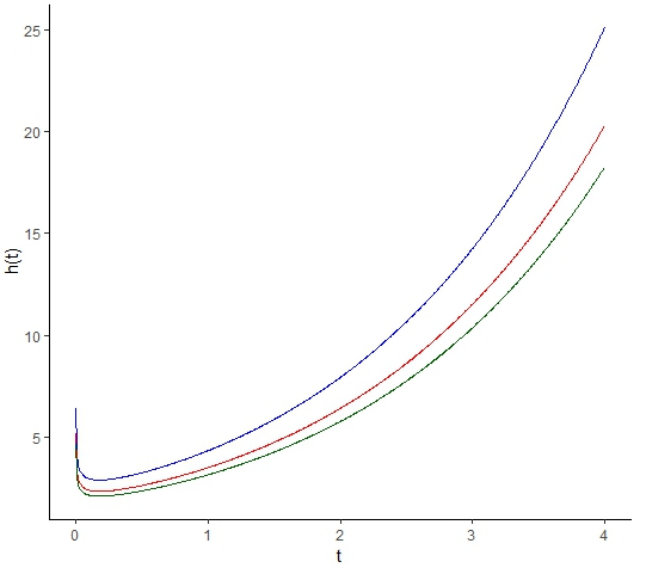

(a) $\mathbf{n}=\mathbf{5 0}, \mathbf{r}=\mathbf{1 0}$

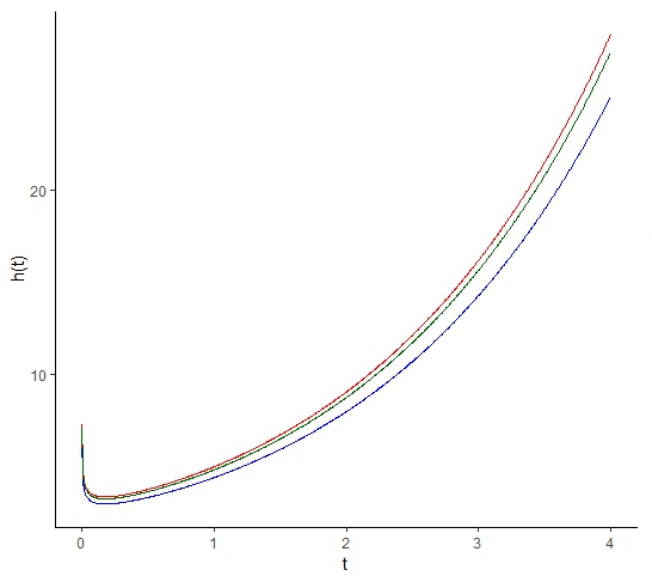

(c) $\mathbf{n}=\mathbf{5 0}, \mathbf{r}=\mathbf{3 0}$

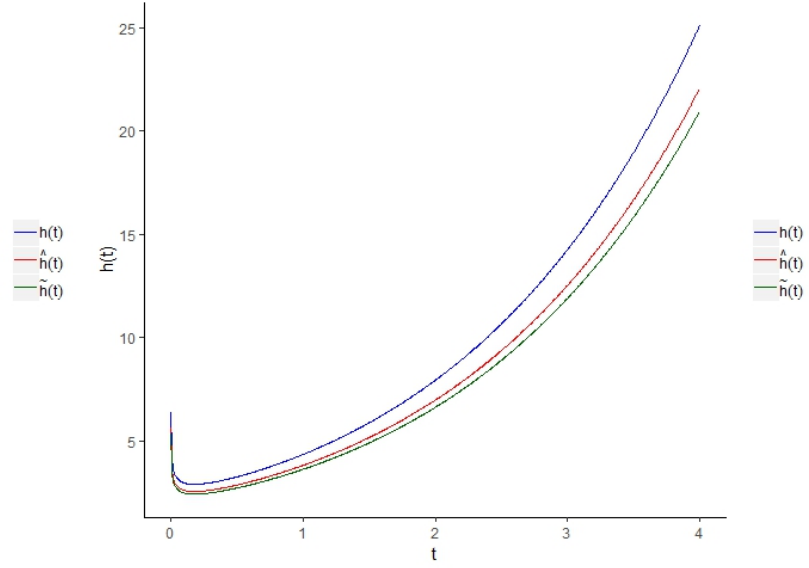

(b) $\mathbf{n}=\mathbf{5 0}, \mathbf{r}=\mathbf{2 0}$

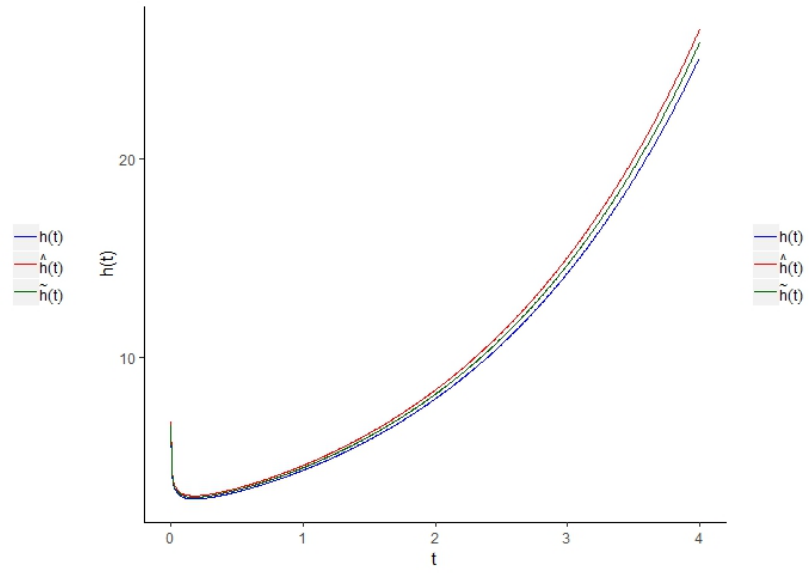

(d) $\mathbf{n}=\mathbf{5 0}, \mathbf{r}=40$

Figure 1. Plots of $h(t)$ and it's estimates against time $\mathbf{t}$

Table 7. UMVUE's and MLE's of $R(t)$ based on the Sampling Scheme of Bartholomew

\begin{tabular}{|c|c|c|c|c|c|c|c|c|c|c|c|}
\hline \multicolumn{2}{|c|}{$t_{\circ}->$} & \multicolumn{2}{|c|}{0.20} & \multicolumn{2}{|c|}{0.45} & \multicolumn{2}{|c|}{0.50} & \multicolumn{2}{|c|}{0.65} & \multicolumn{2}{|c|}{0.80} \\
\hline$t \downarrow$ & $R(t) \downarrow$ & $R(t)$ & $\widetilde{R(t)}$ & $R(t)$ & $R(t)$ & $R(t)$ & $\widetilde{R(t)}$ & $R(t)$ & $\widetilde{R(t)}$ & $R(t)$ & $\widehat{R(t)}$ \\
\hline \multirow{2}{*}{0.25} & \multirow{2}{*}{0.9683} & 0.9704 & 0.9709 & 0.9693 & 0.9694 & 0.9699 & 0.9699 & 0.9717 & 0.9717 & 0.9735 & 0.9735 \\
\hline & & $9 e-04$ & $9 \mathrm{e}-04$ & $2 e-04$ & $2 e-04$ & 0.00012 & 0.00012 & $7 e-05$ & $7 e-05$ & $5 e-05$ & $5 e-05$ \\
\hline \multirow{2}{*}{0.45} & \multirow{2}{*}{0.8938} & 0.8945 & 0.8998 & 0.8974 & 0.8984 & 0.8991 & 0.8999 & 0.9031 & 0.9035 & 0.9107 & 0.9109 \\
\hline & & 0.0102 & 0.0093 & 0.0017 & 0.0017 & 0.0012 & 0.0012 & $6 e-04$ & $6 e-04$ & $6 e-04$ & $6 e-04$ \\
\hline \multirow{2}{*}{0.8} & \multirow{2}{*}{0.6388} & 0.6428 & 0.6991 & 0.6499 & 0.6609 & 0.6597 & 0.6682 & 0.6678 & 0.6724 & 0.6843 & 0.6869 \\
\hline & & 0.0905 & 0.0713 & 0.0132 & 0.0129 & 0.0107 & 0.0107 & 0.0058 & 0.006 & 0.0047 & 0.0049 \\
\hline \multirow{2}{*}{0.9} & \multirow{2}{*}{0.5358} & 0.5485 & 0.6382 & 0.5532 & 0.571 & 0.5504 & 0.5647 & 0.5685 & 0.5761 & 0.5924 & 0.5967 \\
\hline & & 0.1322 & 0.1028 & 0.0209 & 0.0209 & 0.0149 & 0.0149 & 0.0086 & 0.0089 & 0.0068 & 0.0072 \\
\hline
\end{tabular}


From Table 7, it is observed that based on the sampling scheme of Bartholomew, for small values of $t$ and all values of $t_{\circ}$, UMVUE and MLE of $R(t)$ are equally efficient. For large values of $t$ and small values of $t_{\circ}$, MLE is more efficient than UMVUE of $R(t)$. However, for large values of $t_{\circ}$, UMVUE becomes more efficient than MLE of $R(t)$. This result shows the importance of termination time $t_{\circ}$ in the sampling scheme of Bartholomew.

In order to investigate the performance of estimators of $P$ based on the sampling scheme of Bartholomew, we have generated 1000 random samples from each of the populations $X$ and $Y$ with sizes $(n, m)$ from (1) with $\beta_{1}=\beta_{2}=2$ and $\left(\lambda_{1}, \lambda_{2}\right)=(0.5,0.5),(0.5,1),(0.5,1.5)$ and $(0.5,2)$. For each sample corresponding to both the populations by fixing the termination time at $t_{\circ}=t_{\circ}$ and replacing the failure by operating one, values of $r$ (number of failures before time $t_{\circ}$ in $\mathrm{X}$ ) and values of $s$ (number of failures before time $t_{\circ \circ}$ in $\mathrm{Y}$ ) are computed. For $t_{\circ}=t_{\circ \circ}=0.80,1$ and 1.5, we have computed average values of $\widetilde{P}_{I}$ and $\widehat{P}_{I}$ and their corresponding MSE's for $n>m$ and $n<m$ and the results are presented in Tables 8 and 9 respectively.

Table 8. UMVUE's and MLE's of $P$ based on the Sampling Scheme of Bartholomew

\begin{tabular}{|c|c|c|c|c|c|c|c|c|}
\hline \multirow{2}{*}{$\begin{array}{r}\lambda_{1}-> \\
\lambda_{2}-> \\
P-> \\
t_{\circ}=t_{\circ \circ \downarrow}\end{array}$} & & & \multicolumn{2}{|c|}{$\begin{array}{c}1 \\
0.6666667\end{array}$} & \multicolumn{2}{|c|}{$\begin{array}{c}1.5 \\
0.75\end{array}$} & \multicolumn{2}{|c|}{$\begin{array}{c}2 \\
0.8\end{array}$} \\
\hline & $\widetilde{P}$ & $\widehat{P}$ & \multicolumn{2}{|c|}{$\begin{array}{r}\mathbf{0 . 6 6 6 6 6 6 7} \\
\widehat{P} \\
\end{array}$} & \multicolumn{2}{|c|}{$\widehat{P}$} & \multirow[t]{2}{*}{$\widetilde{P}$} & \multirow[t]{2}{*}{$\widehat{P}$} \\
\hline & & & & 0) $>(m$ & & & & \\
\hline \multirow{2}{*}{0.80} & 0.506 & 0.5031 & 0.6357 & 0.6337 & 0.689 & 0.6874 & 0.7219 & 0.7206 \\
\hline & 0.0062 & 0.0062 & 0.0043 & 0.0045 & 0.0061 & 0.0064 & 0.008 & 0.0082 \\
\hline \multirow{2}{*}{1} & 0.5043 & 0.5025 & 0.6035 & 0.6021 & 0.6447 & 0.6435 & 0.6681 & 0.6671 \\
\hline & 0.0024 & 0.0024 & 0.0055 & 0.0057 & 0.0125 & 0.0127 & 0.0186 & 0.0189 \\
\hline \multirow{2}{*}{1.5} & 0.5009 & 0.5 & 0.5389 & 0.5381 & 0.5734 & 0.5726 & 0.6081 & 0.6074 \\
\hline & $4 \mathrm{e}-04$ & $4 \mathrm{e}-04$ & 0.017 & 0.0172 & 0.0319 & 0.0322 & 0.0377 & 0.038 \\
\hline \multicolumn{9}{|c|}{$(n=50)>(m=45)$} \\
\hline \multirow{2}{*}{0.80} & 0.5022 & 0.5015 & 0.632 & 0.6315 & 0.6869 & 0.6865 & 0.7209 & 0.7206 \\
\hline & 0.0052 & 0.0052 & 0.0043 & 0.0043 & 0.0062 & 0.0063 & 0.0079 & 0.008 \\
\hline \multirow[b]{2}{*}{1} & 0.5019 & 0.5014 & 0.6037 & 0.6034 & 0.6441 & 0.6438 & 0.6682 & 0.6679 \\
\hline & 0.0021 & 0.0021 & 0.0055 & 0.0055 & 0.0124 & 0.0125 & 0.0185 & 0.0186 \\
\hline \multirow{2}{*}{1.5} & 0.5006 & 0.5004 & 0.5374 & 0.5372 & 0.5742 & 0.574 & 0.6069 & 0.6067 \\
\hline & $4 \mathrm{e}-04$ & $4 \mathrm{e}-04$ & 0.0172 & 0.0173 & 0.0315 & 0.0316 & 0.038 & 0.0381 \\
\hline
\end{tabular}

Note: 1st and 2nd rows represent the average estimates and MSE's.

From Table 8, for $n>m$, it is observed that for small $m$ when $n=50$, UMVUE of $P$ gives better results than MLE of $P$. As $m$ increases both the estimators are equally efficient. From Table 9, for $n<m$, it is observed that for small $n$ when $m=50$, MLE of $P$ gives better result than UMVUE of $P$. As $m$ increases both the estimators are equally efficient.

To compare the estimates of $h(t)$ with the true value of $h(t)$, we have plotted $h(t)$ and its estimates for $\beta=0.5$, $\lambda=0.8$ and $t_{\circ}=0.15$ (Figure 2). From theory we observe that both UMVUE and MLE of $h(t)$ are same and hence we have plotted only one to represent both.

Since the plot of estimate of $h(t)$ almost overlaps the plot of hazard rate, it establishes the consistency property of estimators.

\subsection{Simulation based on Hypothesis Testing}

In this section, we check the validity of hypotheses developed in Sections 2.3 and 3.2, respectively. For this purpose, we first test the hypothesis, $H_{\circ}: \lambda=\lambda_{\circ}$ against $H_{1}: \lambda \neq \lambda_{\circ}$ based on Type II censoring. We generate a 
Table 9. UMVUE's and MLE's of $P$ based on the Sampling Scheme of Bartholomew

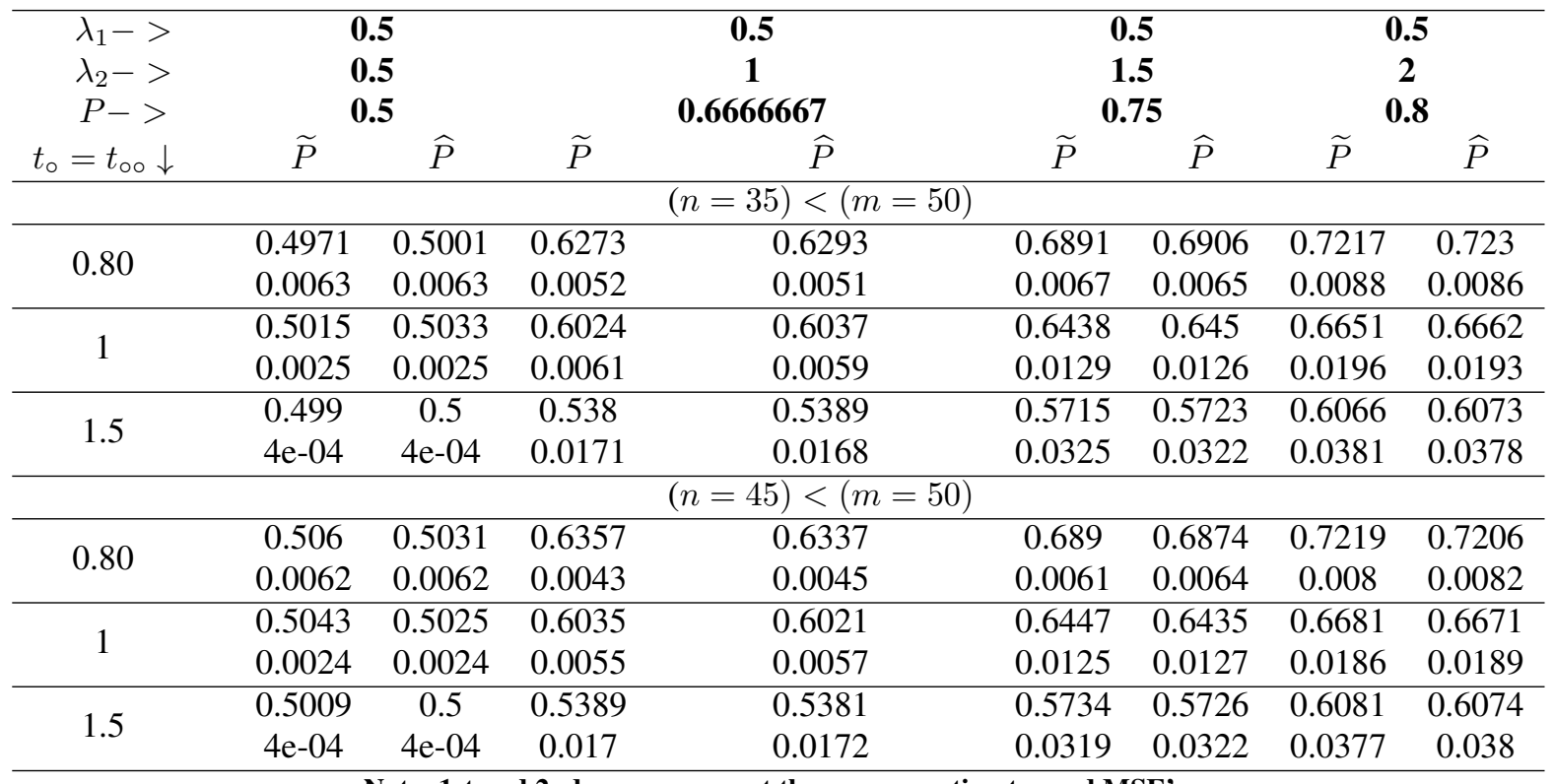

Note: 1st and 2nd rows represent the average estimates and MSE's.

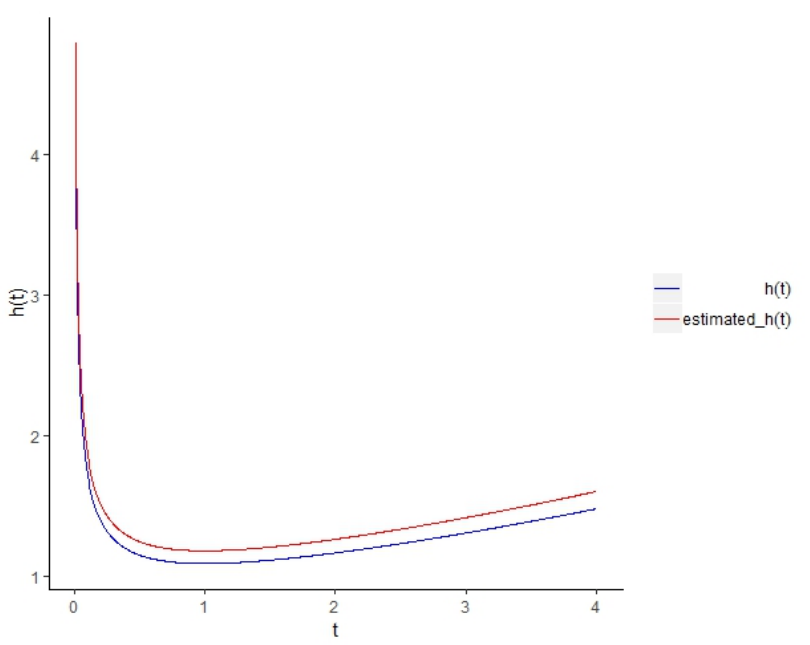

Figure 2. Plots of $\mathbf{h}(\mathbf{t})$ and its estimates under Type I censoring

sample of size $n=50$ from (1) with $\left(\lambda_{1}=0.5, \beta_{1}=2\right)$. The sample is provided below:

Sample 1: $0.0252,0.1352,0.1791,0.2778,0.4252,0.4334,0.4802,0.5379,0.6750,0.6770,0.7204,0.7637$, 0.7894, 0.7997, 0.8187, 0.8498, 0.8920, 0.8930, 0.9050, 0.9191, 0.9201, 0.9414, 0.9881, 1.0000, 1.0028, 1.0196, $1.0255,1.0271,1.0573,1.0882,1.1042,1.1252,1.1408,1.1433,1.1563,1.1610,1.1752,1.1827,1.1901,1.2021$, $1.2043,1.2151,1.2552,1.3069,1.3483,1.3501,1.3513,1.3518,1.3994,1.5697$. 
Now with the help of chi-square table at $\nu=5 \%$ level of significance, for Sample 1 we obtained $k_{\circ}=48.75756$ and $k_{\circ}^{\prime}=95.02318$. As for $r=35$, the value of $S_{35}=85.5457$ is not lying in the critical region, thus we do not reject $H_{\circ}$ at $5 \%$ level of significance. Again considering Sample 1, for testing $H_{\circ}: \lambda \leq \lambda_{\circ}$ against $H_{1}: \lambda>\lambda_{\circ}$ at $5 \%$ level of significance, we obtained $k_{\circ}^{\prime \prime}=51.1393$. As for $r=35$, the value of $S_{(35)}=85.5457$ is not lying in the critical region, thus we do not reject $H_{\circ}$ at $5 \%$ level of significance.

In order to test $H_{\circ}: P=0.6667\left(P_{\circ}\right)$ against $H_{1}: P \neq 0.6667\left(P_{\circ}\right)$ based on Type II censoring scheme, we generated another sample of size $m=60$ from (1.1) with $\left(\lambda_{2}=1, \beta_{2}=2\right)$. The sample is given below:

Sample 2: $0.1625,0.1935,0.1989,0.2128,0.2164,0.2725,0.3750,0.3927,0.3931,0.4140,0.4240,0.4243$, $0.4369,0.4657,0.4705,0.4727,0.5214,0.5269,0.5308,0.5543,0.5777,0.5853,0.5861,0.6032,0.6107,0.6109$, $0.6221,0.6359,0.6404,0.6540,0.6665,0.6773,0.6824,0.7097,0.7152,0.7162,0.7746,0.7791,0.7963,0.8003$, $0.8109,0.8297,0.8338,0.8483,0.8772,0.8823,0.8829,0.8947,0.9100,0.9357,0.9944,0.9952,1.0122,1.0257$, $1.0288,1.1054,1.1166,1.1461,1.1486,1.1805$.

For $r^{\prime}=40$, we get $T_{(40)}=33.2952$. From these samples we get $S_{(35)} / T_{(40)}=2.569314$. Now with the help of F-table at $5 \%$ level of significance, we obtained $k_{2}=1.4183$ and $k_{2}^{\prime}=3.5386$. Hence, in this case we may accept $H_{\circ}$ at $5 \%$ level of significance.

The similar calculations may be done for testing the above hypotheses under the sampling scheme of Bartholomew.

\section{Real Data Analysis}

Now we provide real data analysis based on Type II censoring, when all the parameters of the distribution are unknown, to see how the model works in practice.

We consider the first real data set which was used by [22] (initially taken from [14]) to illustrate the proposed methodology. The data comprise of 50 observations, which represents the quantity of $1000 \mathrm{~s}$ of cycles to failure for electrical appliances in a life test. The data is presented below:

First data set: $\mathrm{x}=(0.014,0.034,0.059,0.061,0.069,0.08,0.123,0.142,0.165,0.21,0.381,0.464,0.479,0.556$, $0.574,0.839,0.917,0.969,0.991,1.064,1.088,1.091,1.174,1.27,1.275,1.355,1.397,1.477,1.578,1.649$, $1.702,1.893,1.932,2.001,2.161,2.292,2.326,2.337,2.628,2.785,2.811,2.886,2.993,3.122,3.248,3.715$, $3.79,3.857,3.912,4.1)$

The second data set was used by [20] (initially taken by [6]). It represents the failure data of a 180 ton rear dump truck. The data shows the number of hours between 128 failures. The data is presented below:

Second data set: $\mathrm{y}=(0.01,0.01,0.01,0.01,0.01,0.02,0.02,0.02,0.02,0.03,0.04,0.06,0.08,0.1,0.1,0.12$, $0.12,0.12,0.13,0.14,0.15,0.15,0.15,0.16,0.16,0.17,0.18,0.18,0.19,0.2,0.21,0.22,0.23,0.25,0.26,0.28$, $0.28,0.3,0.32,0.34,0.36,0.38,0.39,0.41,0.41,0.42,0.43,0.44,0.44,0.45,0.45,0.5,0.53,0.56,0.58,0.58,0.61$, $0.62,0.62,0.62,0.64,0.66,0.7,0.7,0.7,0.72,0.77,0.78,0.78,0.8,0.82,0.83,0.85,0.86,0.96,0.97,0.98,0.99$, $1.05,1.06,1.07,1.18,1.35,1.36,1.42,1.55,1.59,1.65,1.73,1.77,1.79,1.8,1.91,2.09,2.14,2.15,2.15,2.31$, $2.33,2.36,2.43,2.45,2.5,2.51,2.58,2.64,2.68,3.08,3.94,4.12,4.33,4.42,4.53,4.88,4.97,5.11,5.32,5.55$, $6.63,6.89,7.62,11.41,11.76,11.85,12.36,13.22)$

We first apply the KS test to check whether the distribution given at (1) fits the given $\mathrm{X}$ and $\mathrm{Y}$ populations. Fitting the distribution given in (1) to the data $\mathrm{x}$ and $\mathrm{y}$, we obtain the following MLE's of $\left(\lambda_{1}, \beta_{1}\right)$ and $\left(\lambda_{2}, \beta_{2}\right)$ based on complete data sets.

$$
\left(\lambda_{1}, \beta_{1}\right)=(2.3651,0.9371)
$$


and

$$
\left(\lambda_{2}, \beta_{2}\right)=(3.5242,0.6072)
$$

With the help of these Maximum Likelihood Estimators of parameters we apply KS test and conclude that both the data observed for $X(K S=0.0881 ; p=0.7993)$ and the data observed for $Y(K S=0.0857 ; p=0.3119)$ are drawn from (1). Figure 3 and 4 confirms the good fit of (1), for these two data sets.

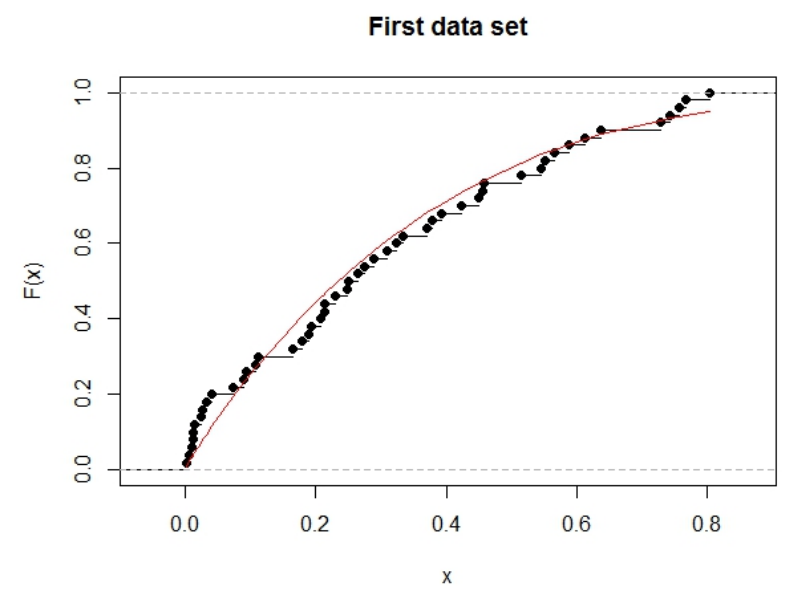

Figure 3. The empirical and theoretical cdf of first data set

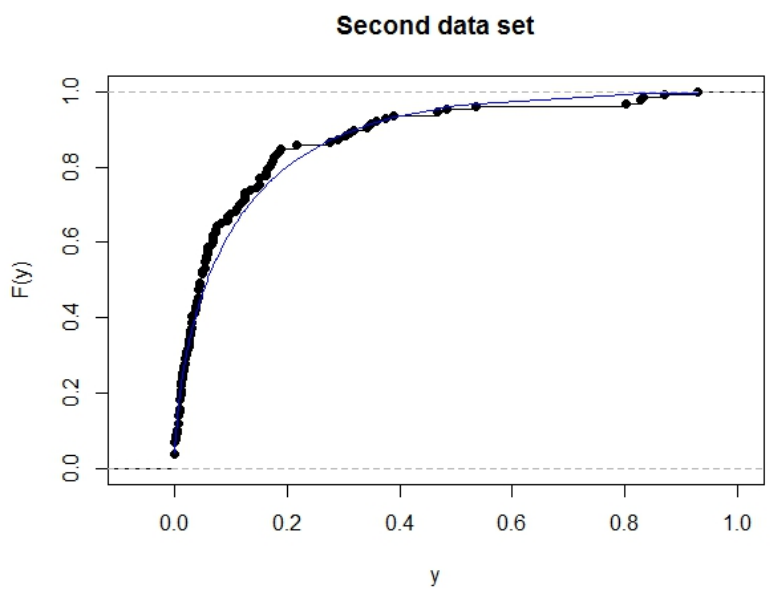

Figure 4. The empirical and theoretical cdf of second data set

In order to obtain the MLE of $R(t)$ and $P$ based on Type II censoring, we first consider $r=30$ lifetimes from $\mathrm{X}$ population and rest 20 observations are considered as censored. Similarly, we consider first $r^{\prime}=90$ lifetimes from Y population and rest 38 observations are considered as censored. Considering Chen distribution as a lifetime model for X-population, for the first $\mathrm{r}$ observations, the MLE's of $\lambda_{1 I I}$ and $\beta_{1 I I}$ comes out to be $\widehat{\lambda_{1 I I}}=2.0992$ and $\widehat{\beta_{1 I I}}=0.8702$. The MLE of $R(t)$ at time point $t=0.15$ is given by $\widehat{R}(t)_{1 I I}=0.6414$. 
Similarly, by considering Chen distribution as a lifetime model for Y-population, for the first $r^{\prime}$ observations, the MLE's of $\lambda_{2 I I}$ and $\beta_{2 I I}$ comes out to be $\widehat{\lambda_{2 I I}}=4.1559$ and $\widehat{\beta_{2 I I}}=0.6560$. The MLE of $R(t)$ at time point $t=0.15$ is given by $\widehat{R}(t)_{2 I I}=0.2497$

To evaluate MLE of $P_{I I}$, we have considered first data set as X Population and second data set as Y population and obtain $\widehat{P}_{I I}=0.4331$. For $\mathrm{X}$ and $\mathrm{Y}$ populations and corresponding to different values of $\mathrm{t}$, we have evaluated MLE of $\mathrm{h}(\mathrm{t})$. Results are plotted in Figure 5.

In particular, for $t=0.15$,

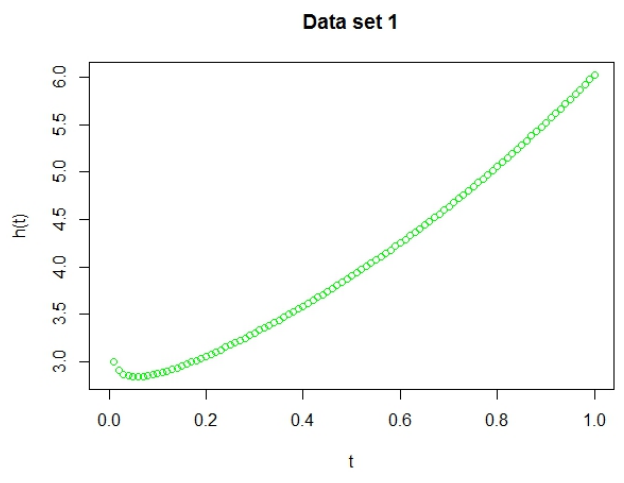

(a) The plot of $h(t)$ against time $t$ for first data set

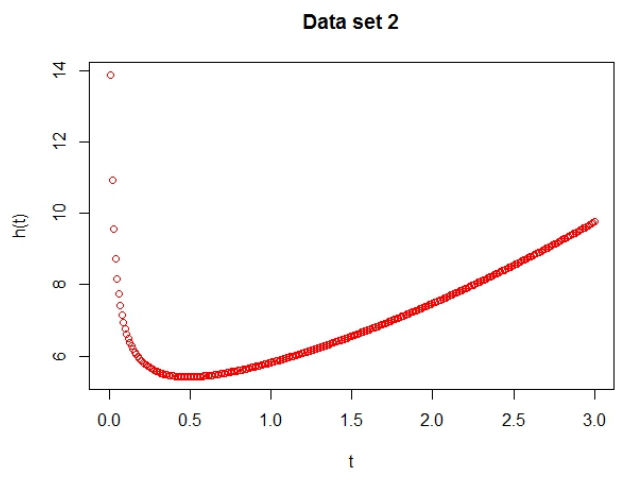

(b) The plot of $h(t)$ against time $t$ for second data set

Figure 5. Plots of $h(t)$ for real life data sets

$$
\widehat{h}_{1 I I}(t)=2.8311
$$

and

$$
\widehat{h}_{2 I I}(t)=6.1841,6.6871 .
$$

From Figure 5, it is clear that the plot of $h(t)$ for both data sets have the bathtub shape.

\section{Concluding Remarks}

In this article, we have developed the estimation procedures for the Chen distribution based on Type II Censoring and Sampling scheme of Bartholomew. Considerations are given to both point and interval estimations. Hypotheses were developed for various parametric functions. The finite sample performance of the UMVUEs and MLEs of reliability functions and other parameters are investigated using extensive Monte Carlo simlation. For Type II censoring, the performance of UMVUE of $\lambda^{q}$ is better than MLE. Also for different values of $t$, the performance of UMVUE of $R(t)$ is better than the performance of MLE of $R(t)$. However, for $t=1$ and beyond, the performance of MLE is better than UMVUE. Moreover, for all values of $\left(r, r^{\prime}\right)$, MLE of $P$ gives better result than the UMVUE of $P$. In case of Sampling scheme of Bartholomew, for small values of $t$ and all values of $t_{\circ}$, UMVUE and MLE of $R(t)$ are equally efficient. But for large values of $t$ and small values of $t_{\circ}$, MLE is more efficient than UMVUE of $R(t)$. However for large values of $t_{\circ}$, UMVUE becomes more efficient than MLE of $R(t)$, thus depicting the importance of termination time $t_{\circ}$ in this scheme. From the study of performance of $P$ it has been observed that for small $\mathrm{m}$ when $n=50$, UMVUE of $P$ gives better result than MLE of $P$. On the other hand, for $n<m$, it is observed that for small $\mathrm{n}$ when $m=50$, MLE of $P$ gives better result than UMVUE of $P$. As $\mathrm{n}$ and $m$ increases both estimators become equally efficient. With the help of Figures 1 and 2 we have established the consistency of estimators of $h(t)$ under both censoring schemes. The real life data examples demonstrate how the proposed estimators of two measures of reliability and confidence ellipsoids can be implemented in practice. 


\section{Acknowledgement}

The authors are grateful to the editor-in-chief and anonymous referee(s) for their valuable suggestions which greatly improved the presentation of this manuscript.

\section{Appendix}

Proof of Lemma 1 Using (1) the joint pdf of $0<X_{(1)} \leq X_{(2)} \leq \ldots \leq X_{(n)}<\infty$ is given by

$$
f\left(x_{(1)}, x_{(2)}, \ldots, x_{(n)} ; \alpha, \beta\right)=n ! \lambda^{n} \beta^{n} e^{\sum_{i=1}^{n} x_{(i)}^{\beta}} \prod_{i=1}^{n} x_{(i)}^{\beta-1} \exp \left(-\lambda \sum_{i=1}^{n}\left(e^{x_{(i)}^{\beta}}-1\right)\right)
$$

Integrating out $x_{(r+1)}, x_{(r+2)}, \ldots, x_{(n)}$ from (A.1) over the region $x_{(r)} \leq x_{(r+1)} \leq \ldots \leq x_{(n)}<\infty$ the joint pdf of $x_{(1)} \leq x_{(2)} \leq \ldots \leq x_{(r)}$ comes out to be

$$
h\left(x_{(1)}, x_{(2)}, \ldots, x_{(r)} ; \lambda, \beta\right)=\frac{n !}{(n-r) !} \lambda^{r} \beta^{r} e^{\sum_{i=1}^{r} x_{(i)}^{\beta-1}} \prod_{i=1}^{r} x_{(i)}^{\beta-1} e^{-\lambda s_{r}}
$$

It follows from (A.2) and Fisher-Neyman factorization theorem (see [19], pp. 347) that, $S_{r}$ is sufficient for the distribution given in (1). Moreover, if we consider the transformation $Z_{i}=(n-i+1)\left\{U_{(i)}-U_{(i-1)}\right\}, i=$ $1,2, \ldots r ; U_{o}=0$, where $U_{(i)}=e^{x_{(i)}^{\beta}}-1$ then $Z_{i}^{\prime} s$ are independent and identically distributed (i.i.d.) rv's, each having exponential distribution with mean life $1 / \alpha$. It is easy to see that $\sum_{i=1}^{r} Z_{i}=S_{r}$. Lemma 1 now follows from the additive property of gamma distribution (see [6], pp. 170). Since the distribution of $S_{r}$ belongs to one parameter exponential family of distributions for known $\beta$, it is also complete (see [19], pp. 347).

Proof of Lemma 2 Let us make the transformations

$$
\left\{\begin{array}{l}
W_{1}=e^{x_{1}^{\beta}}-1, \\
W_{2}=e^{x_{2}^{\beta}}-e^{x_{1}^{\beta}}, \\
\vdots \\
W_{n}=e^{x_{n}^{\beta}}-e^{x_{n-1}^{\beta}}
\end{array}\right.
$$

The pdf of $W_{1}$ is

$$
h\left(w_{1}\right)=n \lambda e^{-n \lambda w_{1}} .
$$

Moreover, $W_{2}, W_{3}, \ldots, W_{n}$ are i.i.d. as $W_{1}$. Using the monotonicity property of $e^{x^{\beta}}-1$, we get

$$
\begin{aligned}
P\left\{N\left(t_{\circ}=r \mid t_{\circ}\right)\right\} & =P\left[X_{(r)} \leq t_{\circ}\right]-P\left[X_{(r+1)} \leq t_{\circ}\right], \\
& =P\left[\left(e^{x_{(r)}^{\beta}}-1\right) \leq\left(e^{t_{\circ}^{\beta}}-1\right)\right]-P\left[\left(e^{x_{(r-1)}^{\beta}}-1\right) \leq\left(e^{t_{\circ}^{\beta}}-1\right)\right] .
\end{aligned}
$$

Using (A.3) and (A.4), we get

$$
\begin{aligned}
P\left\{N\left(t_{\circ}=r \mid t_{\circ}\right)\right\} & =P\left[W_{1}+W_{2}+\ldots+W_{r} \leq\left(e^{t_{\circ}^{\beta}}-1\right)\right] \\
& -P\left[W_{1}+W_{2}+\ldots+W_{r-1} \leq\left(e^{t_{\circ}^{\beta}}-1\right)\right] .
\end{aligned}
$$

From the additive property of exponentially distributed rvs (see [7], pp. 170), $U=n \lambda \sum_{i=1}^{r} W_{i}$ follows gamma distribution with pdf:

$$
h(u)=\frac{1}{\Gamma(r)} u^{r-1} e^{-u}, u>0 .
$$


Using (A.5) and a result of ([17], pp. 244) we obtain from (A.4) that

$$
\begin{aligned}
P\left\{N\left(t_{\circ}=r \mid t_{\circ}\right)\right\} & =\frac{1}{\Gamma(r+1)} \int_{e^{t_{\circ}^{\beta}}-1}^{\infty} e^{-u} u^{r} d u-\frac{1}{\Gamma(r)} \int_{e^{t_{\circ}^{\beta}}-1}^{\infty} e^{-u} u^{r-1} d u, \\
& =\exp \left(-n \lambda\left(e^{t_{\circ}^{\beta}}-1\right)\right)\left\{\sum_{j=0}^{r} \frac{\left[n \lambda\left(e^{t_{\circ}^{\beta}}-1\right)\right]^{j}}{j !}\right\} \\
& -\exp \left(-n \lambda\left(e^{t_{\circ}^{\beta}}-1\right)\right)\left\{\sum_{j=0}^{r-1} \frac{\left[n \lambda\left(e^{t_{\circ}^{\beta}}-1\right)\right]^{j}}{j !}\right\} .
\end{aligned}
$$

and the lemma follows.

\section{REFERENCES}

1. E. A. Ahmed, Bayesian estimation based on progressive type II censoring from two-parameter bathtub-shaped lifetime model: An Markov chain Monte Carlo approach, , vol. 41, pp. 752-768, 2014.

2. A. Algarni, A. M. Almarashi, H. Okasha, and H. K. T. NG, E-Bayesian Estimation of Chen Distribution Based on Type-I Censoring Scheme, Entropy, vol. 22, no. 636, 2020.

3. D. J. Bartholomew, The sampling distribution of an estimate arising in life testing, Technometrics, vol. 5, pp. 361-374, 1963.

4. A. Chaturvedi, and S. K. Tomer, UMVU estimation of the reliability function of the generalized life distributions, Statistical Papers, vol. 44, no. 3, pp. 301-313, 2003.

5. Z. Chen, A new two-parameter lifetime distribution with bathtub shape or increasing failure rate function, Statistics and Probability Letters, vol. 49, pp. 155-161, 2000.

6. J. L. Coetzeee, Reliability degradation and the equipment replacement problem, Proceedings of the International Conference of Maintenance Societies (ICOMS '96), paper. 21, Melbourne, Australia.

7. N. L. Johnson, and S. Kotz, Continuous Univariate Distributions, Volume 2, Wiley Series in Probability and Statistics, 1970.

8. T. Kayal, Y. M. Tripathi, S. Dey, and S. Wu, On estimating the reliability in a multicomponent stress-strength model based on Chen distribution, Communications in Statistics - Theory and Methods, 2019.

9. T. Kayal, Y. M. Tripathi, D. Kundu, and M. K. Rastogi, Statistical Inference of Chen Distribution Based on Type I Progressive Hybrid Censored Samples, Statistics, Optimization and Information Computing, 2019.

10. T. Kayal, Y. M. Tripathi, D. P. Singh, and M. K. Rastogi, Estimation and prediction for Chen distribution with bathtub shape under progressive censoring, Journal of Statistical Computation and Simulation, vol. 87, pp. 348-366, 2017.

11. M. J. S. Khan, and A. Sharma, Generalized order statistics from Chen distribution and its characterization, Journal of Statistics and Applied Probability, vol. 5, pp. 123-128, 2016.

12. I. Kinachi, K. Karakaya, Y. Akdogan, and C. Kus, Bayesian estimation for discrete Chen distribution, Ekim, vol. 42, pp. 144-148, 2016.

13. C. Lai, M. Xie, and D. Murthy, A modified Weibull distribution, IEEE Transactions on Reliability, vol. 52, pp. 33-37, 2003.

14. J. F. Lawless, Statistical models and methods for lifetime data, Wiley, Hoboken, 362, 2011.

15. E. L. Lehmann, Testing Statistical Hypotheses, John Wiley and Sons, New York, 1959.

16. A. W. Marshall, and I. Olkin, A new method for adding a parameter to a family of distributions with application to the exponential and Weibull families, vol. 84, pp. 641-652, 1997.

17. J. K. Patel, C. H. Kapadia, and D. B. Handbook of Statistical Distributions, Marcel Dekker, New York, 1976.

18. M. K. Rastogi, Y. M. Tripathi, and S. J. Wu, Estimating the parameters of a bathtub-shaped distribution under progressive type-II censoring, Journal of Applied Statistics, vol. 39, pp. 2389-2411, 2012.

19. V. K. Rohatgi, and A. K. Md. E. Saleh, An introduction to probability and statistics, Wiley, New York, 2012.

20. X. Wang, C. Yu, and and Y. Lia, New finite interval lifetime distribution model for fitting bathtub-Shaped failure rate curve Problems in Engineering, Article ID 954327, http://dx.doi.org/10.1155/2015/954327, 2015.

21. S. J. Wu, Estimation of the two parameter bathtub-shaped lifetime distribution with progressive censoring, Journal of Applied Statistics, vol. 35, pp. 1139-1150, 2008.

22. F. Yousaf, S. Ali, and I. Shah, Statistical Inference for the Chen Distribution Based on Upper Record Values, Annals of Data Science, vol. 6, pp. 831-858, 2019. 